Total organic carbon and Rock Eval pyrolysis data and analysis for the Consolidated 0il Iniskin Unit Zappa No. 1 well (See GMC Data Report No. 83), and for the Consolidated 0il Iniskin Unit Beal No. 1 well. 
Iniskin Wel1' (930' to 11200'. samples 6436-001 to -014)

\section{Organic Richness}

From 930" to 5090' TOC values of 0.30 to 0.66 wt.x generally reflect poor source rock organic carbon quantity. A sample at 6240-6270' with TOC 0.87 wt.k has fair quantity, and from 6500' to $9080^{\prime}$ threg samples with values of 1.01 to 1.41 wt. $\%$ marginally qualify as good source quantities. The deepest sample, at 11170-11200\%. has poor quantity $(0.28 \mathrm{wt}, \mathrm{x})$.

\section{Thermal Maturity}

Based on $T_{\max }$ values for samples from 1430' to $4980^{\prime}$ ( $T_{\max } 435-438^{\circ} \mathrm{C}$ ), maturation varies from 1 mmature to marginally mature (early oil window). Marginally detectable $S_{1}$ values $(0.10)$ improve slightly for samples at $5060^{\prime}$. A shoulder on the $S_{2}$ peak of the sample at 6500-6550" accounte for the break in the downhole increasing trend of $T_{m a x}$. The shoulder could reflect either early generated bitumen or contamination, which can reduce $T_{\max }$.

\section{Quantity of Organic Matter}

At this early stage of maturity the low Hydrogen Indices (28-149) suggest a marginally gas prone source rock to non source rock quality (Type III-Type IV kerogen).

Iniskin Beal \#1 (480' to 8760', samples 6438-023 to -041)

Organic Richness

Poor to fair TOC from 480' to $5120^{\prime}(0.46-0.55$ wt. $x)$. good from 6510' to 8040' $(0.99-1.55$ wt. $x)$, fair from $8520^{\prime}$ to $8760^{\prime}(0.55-0.90$ wt. $x)$.

\section{Thernal Maturity}

Glear unimodal $S_{2}$ peaks have yielded apparently reliable $T_{\max }$ values throughout the well. They vary fairly consistently with depth from marginally mature at the surface $\left(435-440^{\circ} \mathrm{C}\right)$ to well within the 011 window for the deepest samples (up to $448^{\circ} \mathrm{C}$ ). This is supported by increasing $S_{1}$ values and Production Indices up to 0.29 .

\section{Quality of Organic Matter}

No ail prone sample was apparent as the HI never significantly exceeded the HI=150 reguired to characterize a mixed oil and gas prone source. Several samples. however, had values near 150 and considering their having been in the oil window these values could have been reduced by early generation and expulsion.

These data correlate well with the "Iniskin well". 
TABLE II

Results of Total Organic Carbon Analysis and Rock-Eval Pyrolysis

\begin{tabular}{lccccccccc}
$\begin{array}{l}\text { Sample } \\
\text { Number }\end{array}$ & $\begin{array}{c}\text { Depth } \\
(\mathrm{ft})\end{array}$ & $\begin{array}{c}\text { TOC } \\
(\mathrm{Wt} . \%)\end{array}$ & $\begin{array}{c}\mathrm{S} 1 \\
(\mathrm{mg} / \mathrm{g})\end{array}$ & $\begin{array}{c}\mathrm{S} 2 \\
(\mathrm{mg} / \mathrm{g})\end{array}$ & $\begin{array}{c}\mathrm{S} 3 \\
(\mathrm{mg} / \mathrm{g})\end{array}$ & $\begin{array}{l}\text { Tmax } \\
\left({ }^{\circ} \mathrm{C}\right)\end{array}$ & $\begin{array}{c}\text { Production } \\
\text { Index }\end{array}$ & $\begin{array}{c}\text { Hydrogen } \\
\text { Index }\end{array}$ & $\begin{array}{c}\text { Oxygen } \\
\text { Index }\end{array}$ \\
\hline
\end{tabular}

Iniskin Well:

\begin{tabular}{|c|c|c|c|c|c|c|c|c|c|}
\hline $6436-001$ & $930-960$ & 0.30 & --- & $\cdots-$ & -- & $-\cdots$ & -- & -- & -- \\
\hline $6436-002$ & $1430-1460$ & 0.66 & 0.15 & 0.51 & 0.35 & 435 & 0.23 & 78 & 53 \\
\hline $6436-003$ & $2100-2130$ & 0.50 & $<0.10$ & 0.14 & 0.47 & 438 & --- & 28 & 93 \\
\hline $643 G-004$ & $2160-2190$ & 0.57 & $<0.10$ & 0.20 & 0.29 & 438 & --- & 35 & 51 \\
\hline $6436-005$ & $3290-3320$ & 0.54 & $<0.10$ & 0.36 & 0.47 & 434 & $-\cdots$ & 67 & 86 \\
\hline $6436-006$ & $4520-4540$ & 0.53 & $<0.10$ & 0.47 & 0.36 & 438 & -- & 88 & 68 \\
\hline $6436-007$ & $4950-4980$ & 0.46 & $<0.10$ & 0.33 & 0.38 & 438 & --- & 72 & 82 \\
\hline $6436-008$ & $5060-5090$ & 0.58 & 0.11 & 0.65 & 0.37 & 441 & 0.15 & 112 & 64 \\
\hline $6436-009$ & $6240-6270$ & 0.87 & 0.10 & 1.01 & 0.30 & 442 & 0.09 & 116 & 34 \\
\hline $6436-010$ & $6500-6550$ & 1.41 & 0.13 & 1.50 & 0.72 & 438 & 0.08 & 107 & 51 \\
\hline $6436-011$ & $8180-8210$ & 1.01 & 0.23 & 1.06 & 0.34 & 442 & 0.18 & 105 & 33 \\
\hline $6436-012$ & $9060-9080$ & 1.11 & 0.36 & 1.65 & 0.30 & 441 & 0.18 & 149 & 27 \\
\hline $6436-013$ & $10370-10390$ & 0.35 & - & $\cdots$ & $-\infty$ & --- & -- & --- & --- \\
\hline $6436-014$ & $11170-11200$ & 0.28 & --- & $\cdots$ & -- & --- & --- & $\cdots$ & --- \\
\hline
\end{tabular}

Well: Iniskin Beal \#1

\begin{tabular}{|c|c|c|c|c|c|c|c|c|c|}
\hline $6438-023$ & $480-570$ & 0.58 & 0.10 & 0.56 & 0.16 & 438 & 0.15 & 96 & 28 \\
\hline $6438-024$ & $930-1020$ & 0.75 & $<0.10$ & 0.90 & 0.13 & 436 & $m$ & 120 & 17 \\
\hline $6438-025$ & $1530-1620$ & 0.53 & $<0.10$ & 0.28 & 0.14 & 435 & $-\infty$ & 53 & 26 \\
\hline $6438-026$ & $1970-2030$ & 0.55 & $<0.10$ & 0.22 & 0.10 & 440 & -- & 41 & 18 \\
\hline $6438-027$ & $2470-2500$ & 0.79 & $<0.10$ & 1.10 & $<0.10$ & 436 & $\cdots$ & 140 & $\cdots$ \\
\hline $6438-028$ & $2990-3020$ & 0.56 & $<0.10$ & 0.28 & $<0.10$ & 442 & $-\infty$ & 50 & $-n$ \\
\hline $6438-029$ & $3500-3530$ & 0.66 & $<0.10$ & 0.87 & $<0.10$ & 441 & $-\infty$ & 131 & $-\infty$ \\
\hline $6438-042$ & $3530-3590$ & 0.63 & $<0.10$ & 0.60 & $<0.10$ & 443 & - & 95 & $\cdots$ \\
\hline $6438-030$ & $3810-3840$ & 0.46 & $<0.10$ & 0.38 & $<0.10$ & 439 & $\cdots$ & 83 & $\infty$ \\
\hline $6438-031$ & $5070-5100$ & 0.55 & $<0.10$ & 0.44 & $<0.10$ & 445 & 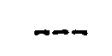 & 80 & $-\infty$ \\
\hline $6438-032$ & $5590-5620$ & 0.55 & $<0.10$ & 0.59 & 0.11 & 441 & -- & 108 & 19 \\
\hline $6438-033$ & $5930-5960$ & 0.85 & 0.15 & 0.96 & 0.12 & 443 & 0.14 & 113 & 14 \\
\hline $6438-034$ & $6510-6530$ & 1.55 & 0.50 & 2.41 & $<0.10$ & 444 & 0.17 & 156 & $m$ \\
\hline $6438-035$ & $7000-7020$ & 1.18 & 0.31 & 1.76 & $<0.10$ & 446 & 0.15 & 149 & $-\infty$ \\
\hline $6438-036$ & $7500-7520$ & 1.07 & 0.24 & 1.44 & 0.10 & 446 & 0.14 & 135 & 10 \\
\hline $6438-037$ & $8020-8040$ & 0.99 & 0.22 & 1.25 & 0.16 & 445 & 0.15 & 126 & 16 \\
\hline $6438-038$ & $8520-8540$ & 0.67 & 0.14 & 0.73 & 0.11 & 445 & 0.16 & 109 & 17 \\
\hline $6438-039$ & $8995-9020$ & 0.70 & 0.28 & 0.69 & 0.42 & 440 & 0.29 & 98 & 59 \\
\hline $6438-040$ & $9380-9410$ & 0.55 & 0.18 & 0.58 & 0.17 & 444 & 0.24 & 106 & 31 \\
\hline $6438-041$ & $8740-8760$ & 0.90 & 0.34 & 1.09 & 0.16 & 448 & 0.24 & 121 & 18 \\
\hline
\end{tabular}




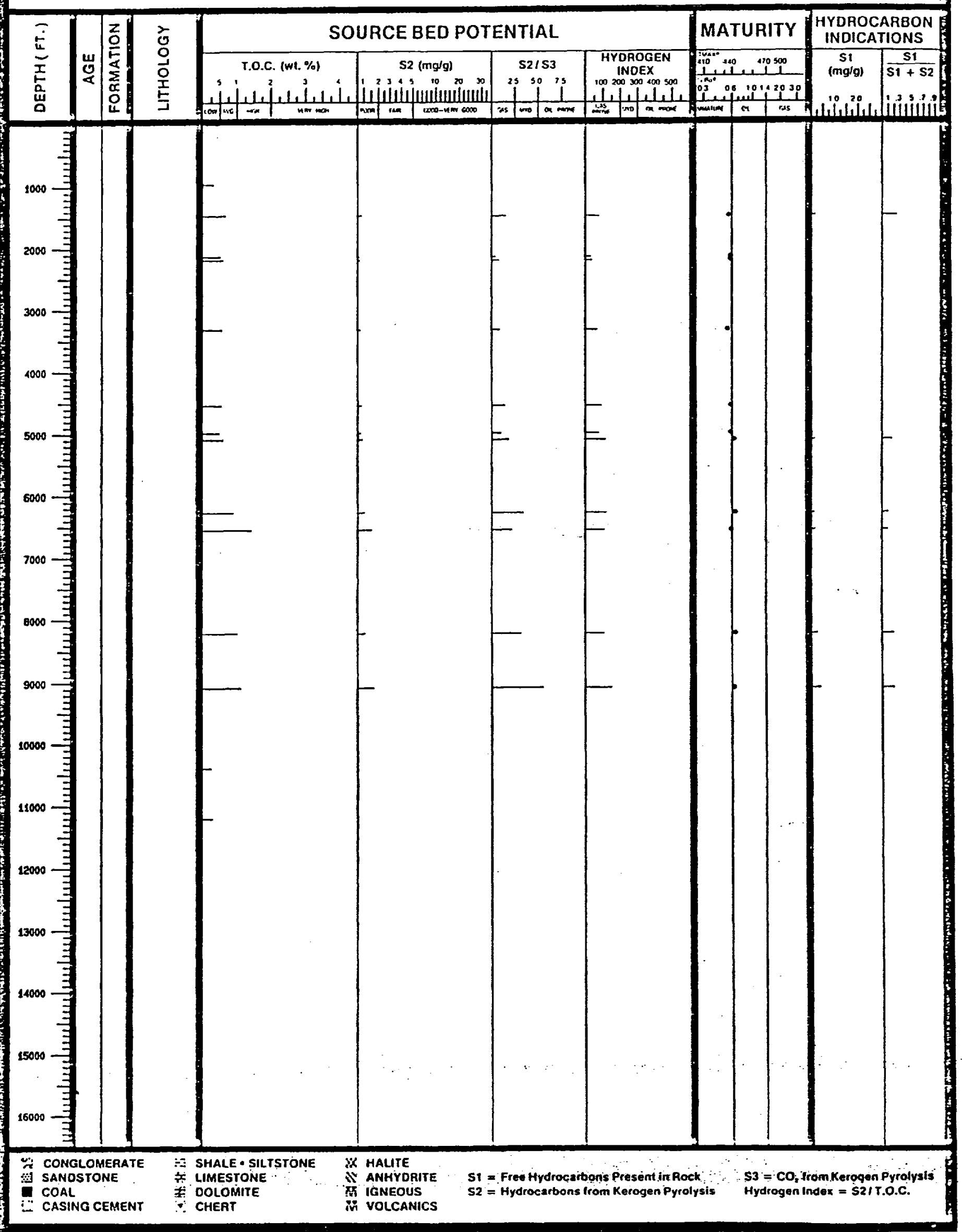




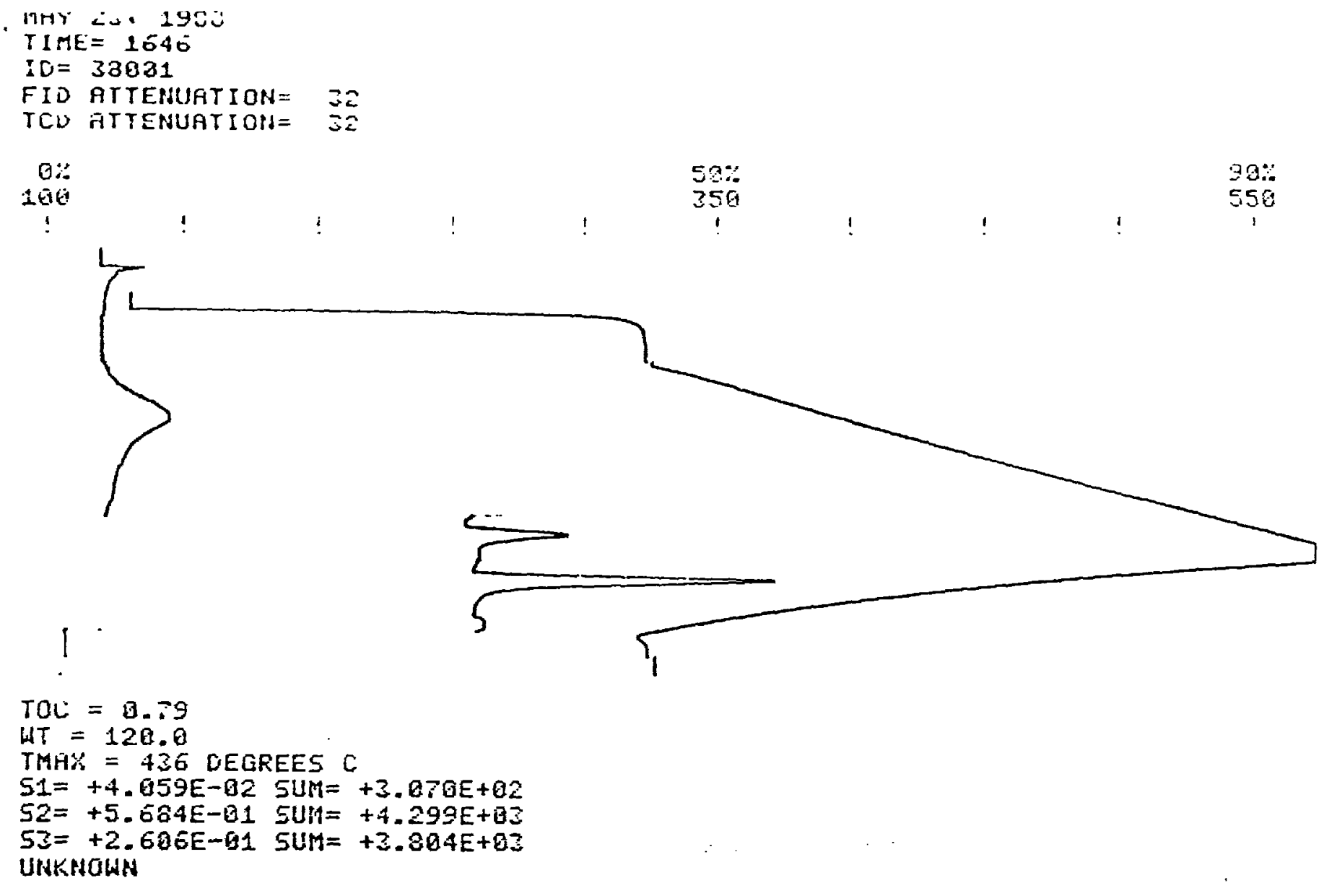

Mค' 23,1988

GEOCON ROCK EHAL II

TIHE $=1712$

ID $=002$

FIU ATTENUATIOH $=32$

TCD ATTENUATION= 32

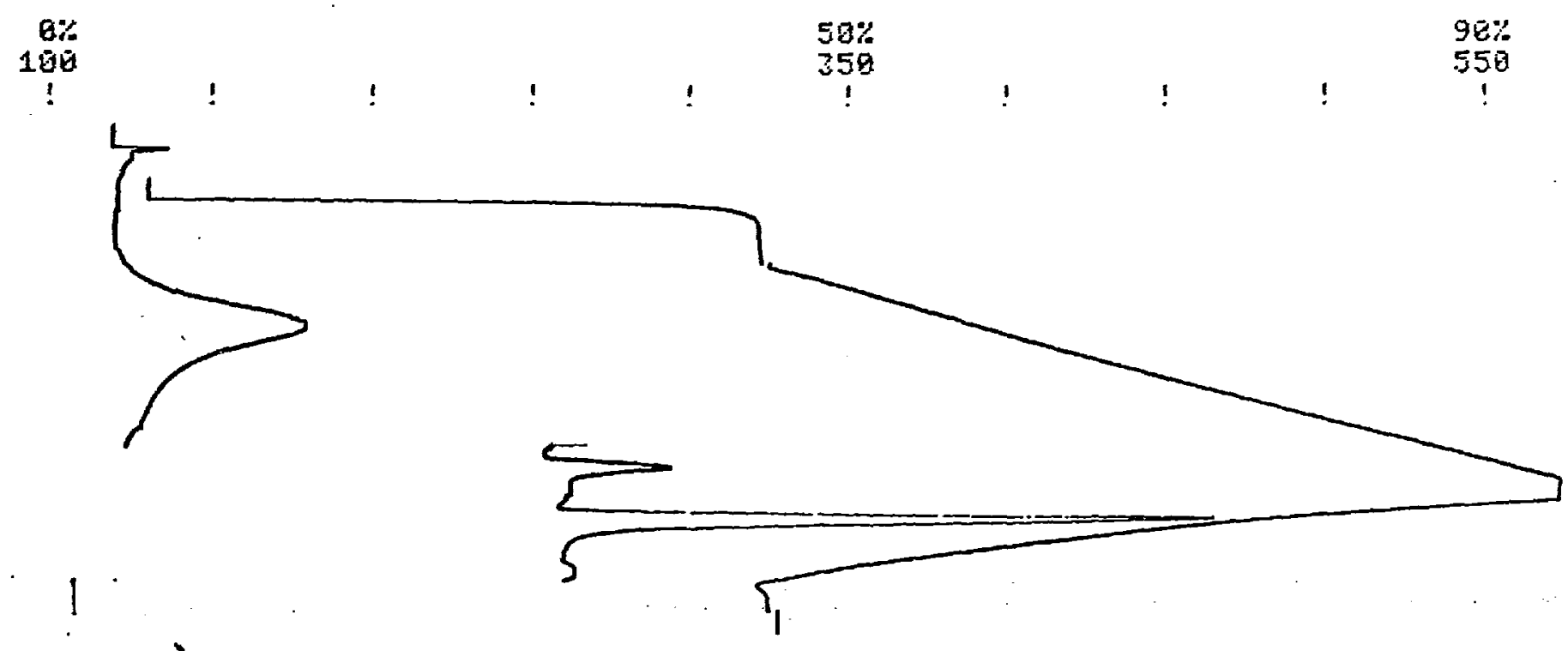

$\mathrm{rac}=1.52$

$H T=119.6$

TMAX $=433$ DEGREES $C$

$51=+3.794 \mathrm{E}-02$ SUM $=+2.860 \mathrm{E}+02$

$S 2=+1.372 E+00$ SUM $=+1.034 E+04$

$S Z=+4.961 E-01$ SUM $=+E .650 E+23$

UNKHOHN 
TIME $=173 a$

$I D=002$

FIU ATTENUAT IOH= $3 \hat{a}$

TCU ATTERUATIO: $=32$

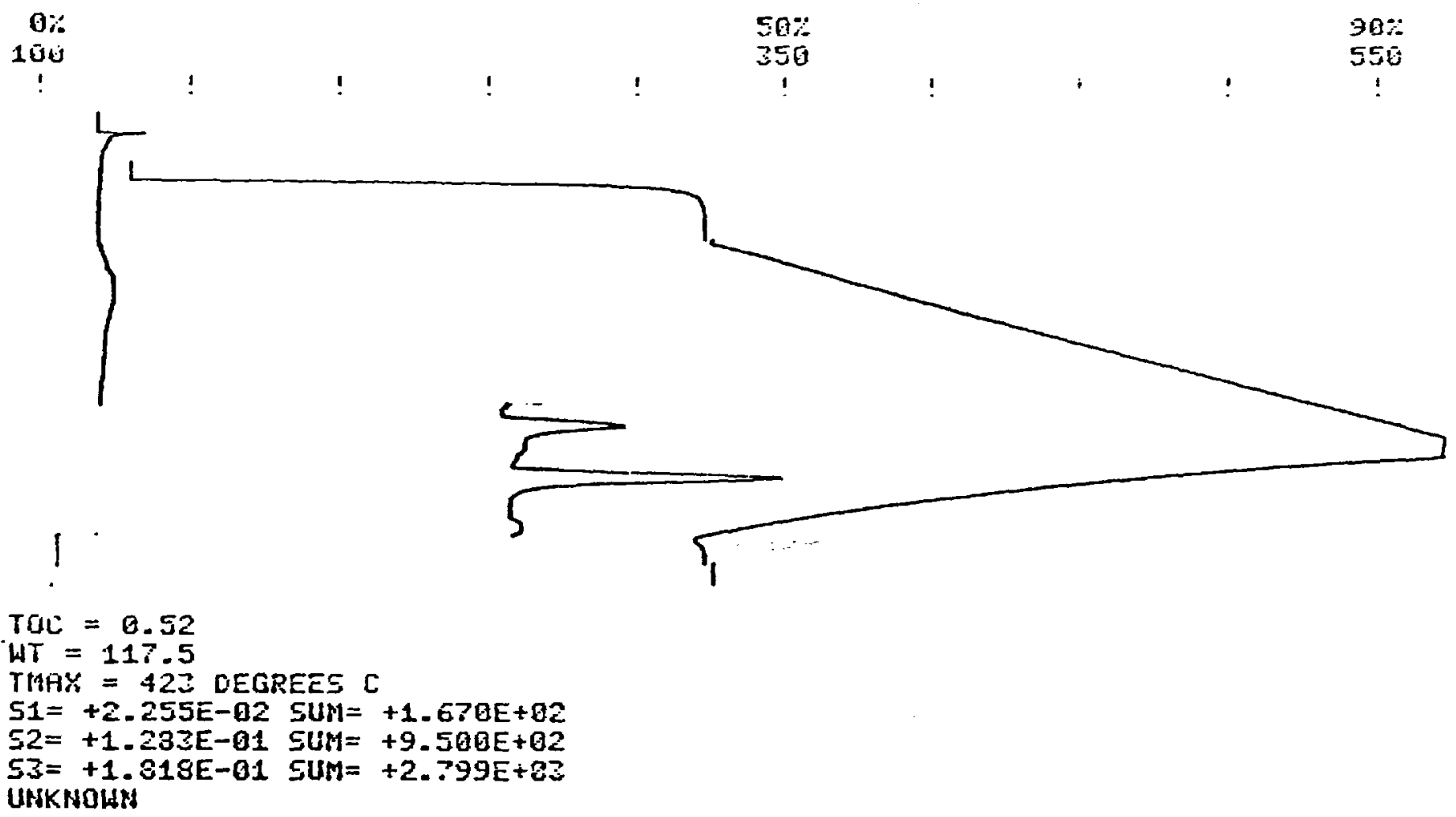

MAY 23, 1988

GEOCOM ROCK EYAL II

TIME $=1305$

$I D=004$

FID ATTEMUATION= 32

TCD ATTENUATION: 32

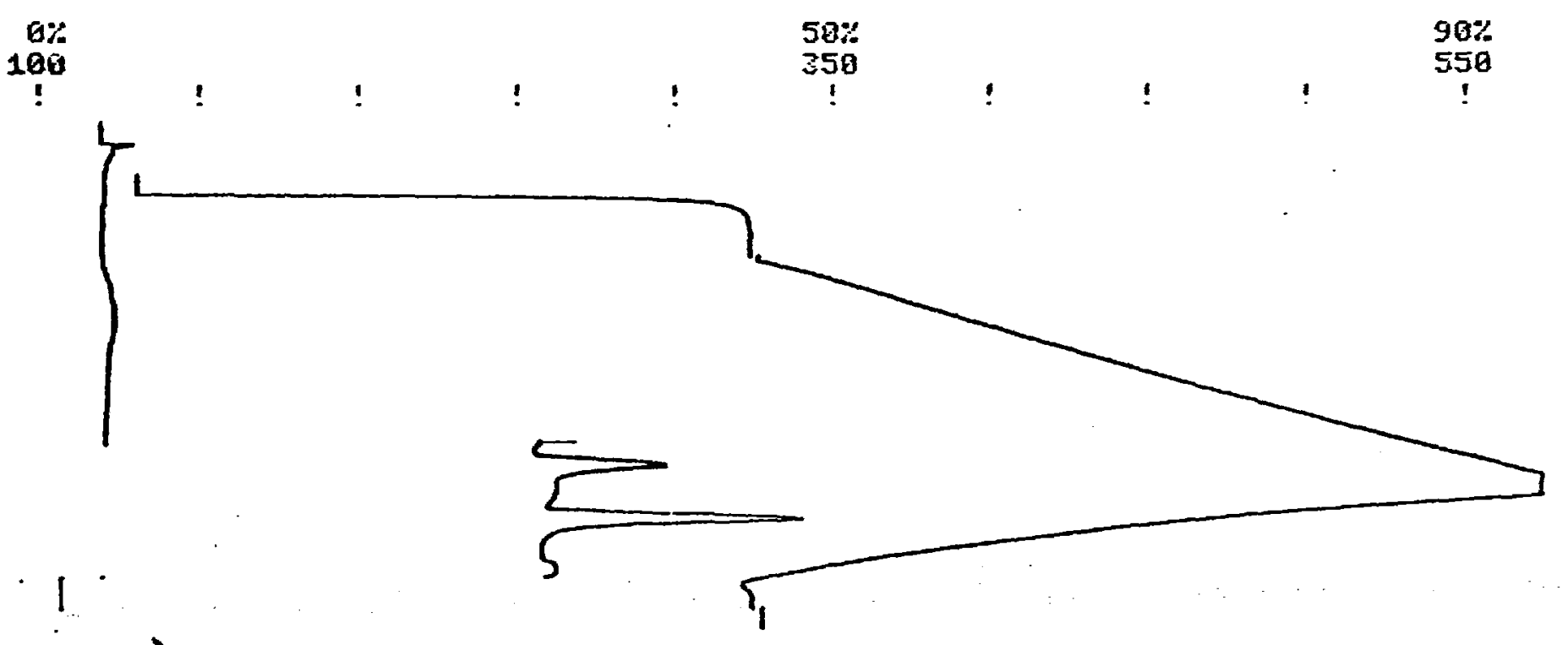

TOC $=0.44$

WT $=121.7$

THHX $=416$ CEGREES $\mathrm{C}$

$S 1=+1.525 E-02$ SUM $=+1.170 E+02$

$S 2=+3.540 E-02 \cdot S U M=+6.550 E+02$

$\mathrm{S} 3=+1.502 \mathrm{E}-01 \mathrm{SUH}=+2.46 E \mathrm{E}+03$ 
MAY 23: 1983

UEULUS ruLr EVML 1

TIME $=1831$

$I D=005$

FID ATTENUATION= 32

TCU ATTENUATICH $=32$

$\theta:$

100

10

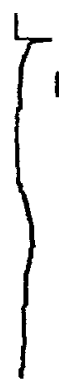

I

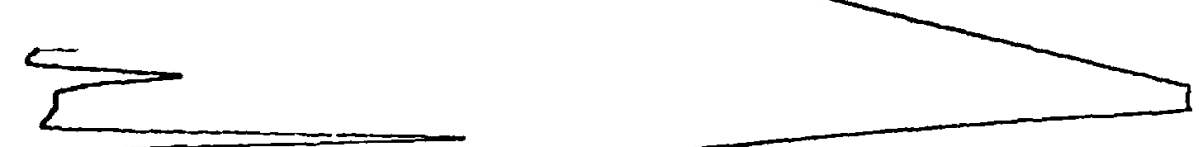

$T O L=8.56$

$W T=120.0$

TMAX $=419$ DEGREES $C$

$S 1=+1.494 E-025 U M=+1.130 E+02$

$52=+8.833 \mathrm{E}-02 \mathrm{SUM}=+6.620 \mathrm{E}+02$

$53=+2.427 E-61$ SUM $=+2.586 E+Q 3$

UNKMOUH

HFY 23, 1988

GEOCOM ROCK EYRL II

TIME $=185 ?$

$I D=006$

FID RTTENUATION $=32$

TCD ATTENUATION $=32$

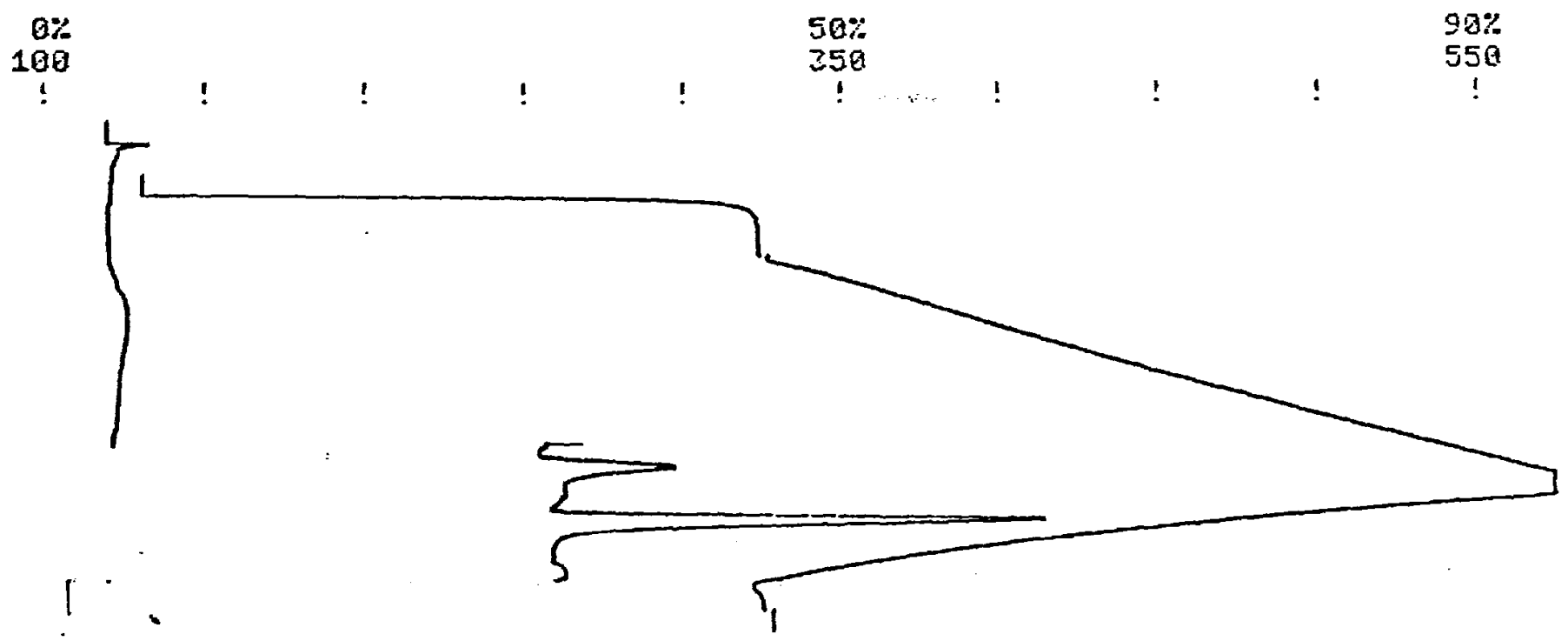

$\mathrm{TOC}=0.69$

$H T=126.0$

TMHX $=426$ DEGREES C

$S_{1}=+1.996 \mathrm{E}-02$ SUM $=+1.516 \mathrm{E}+02$

$S 2=+1.848 E-01$ SUM $=+1.393 E+B 3$

$S 3=+3.399 E-01$ SUM $=+4.769 E+03$

IAKHOUNI 
MAr 23, 1988

GECCON ROCY EYAL I:

TIME $=1324$

$I D=B 6 r$

FIU ATTENUATICH $=\mathrm{gz}$

TCO ATTENUATION= 32

6:2

100

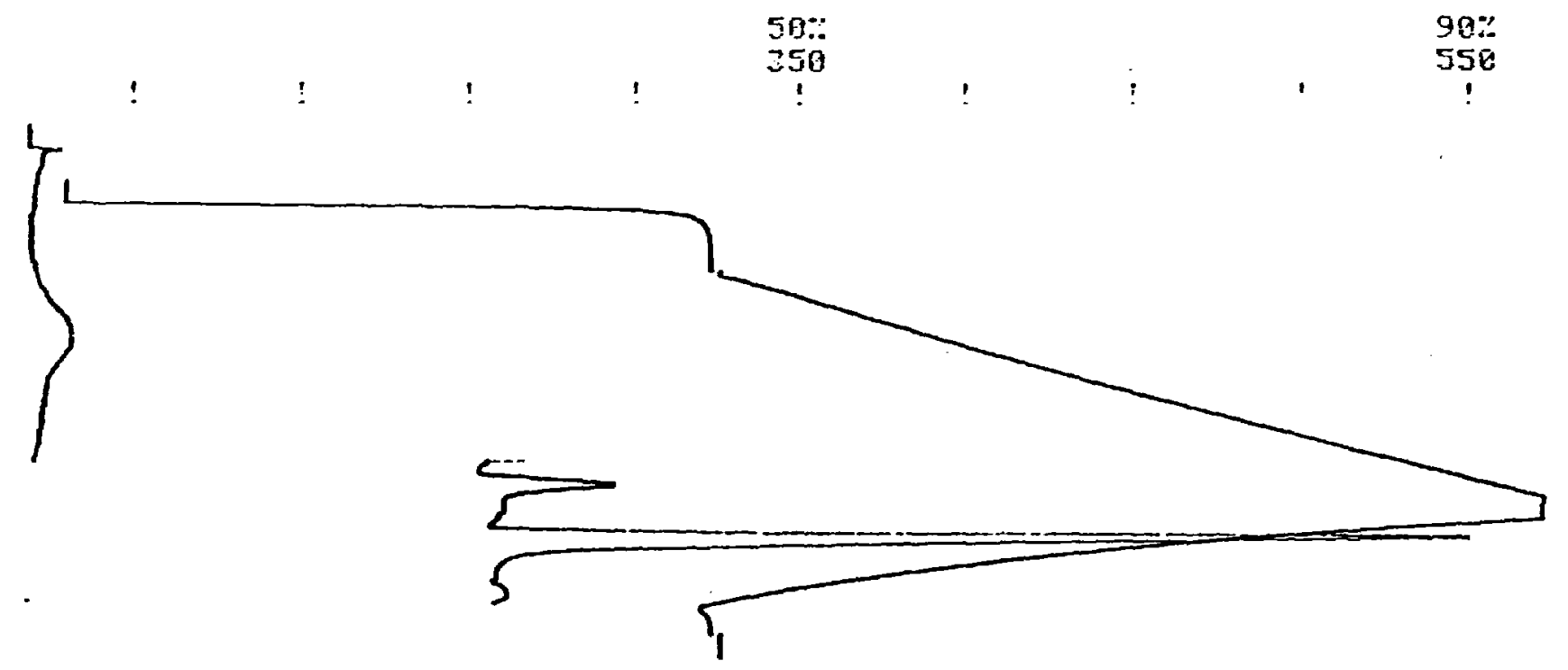

TUL $=0.89$

HT $=120.7$

TMHR $=423$ DEGREES C

$51=+2.513 E-025 U M=+2.140 E+02$

$52=+3.544 E-015 U H=+2.696 E+03$

$S_{3}=+7.174 E-01$ SUH $=+9.416 E+9 ?$

UNKNOWH

MAY 23, 1930

GEOCOM POCK EYAL II

TIME $=1950$

$I D=003$

FID RTTENUATIGH $=32$

TCO ATTENUATION= 32

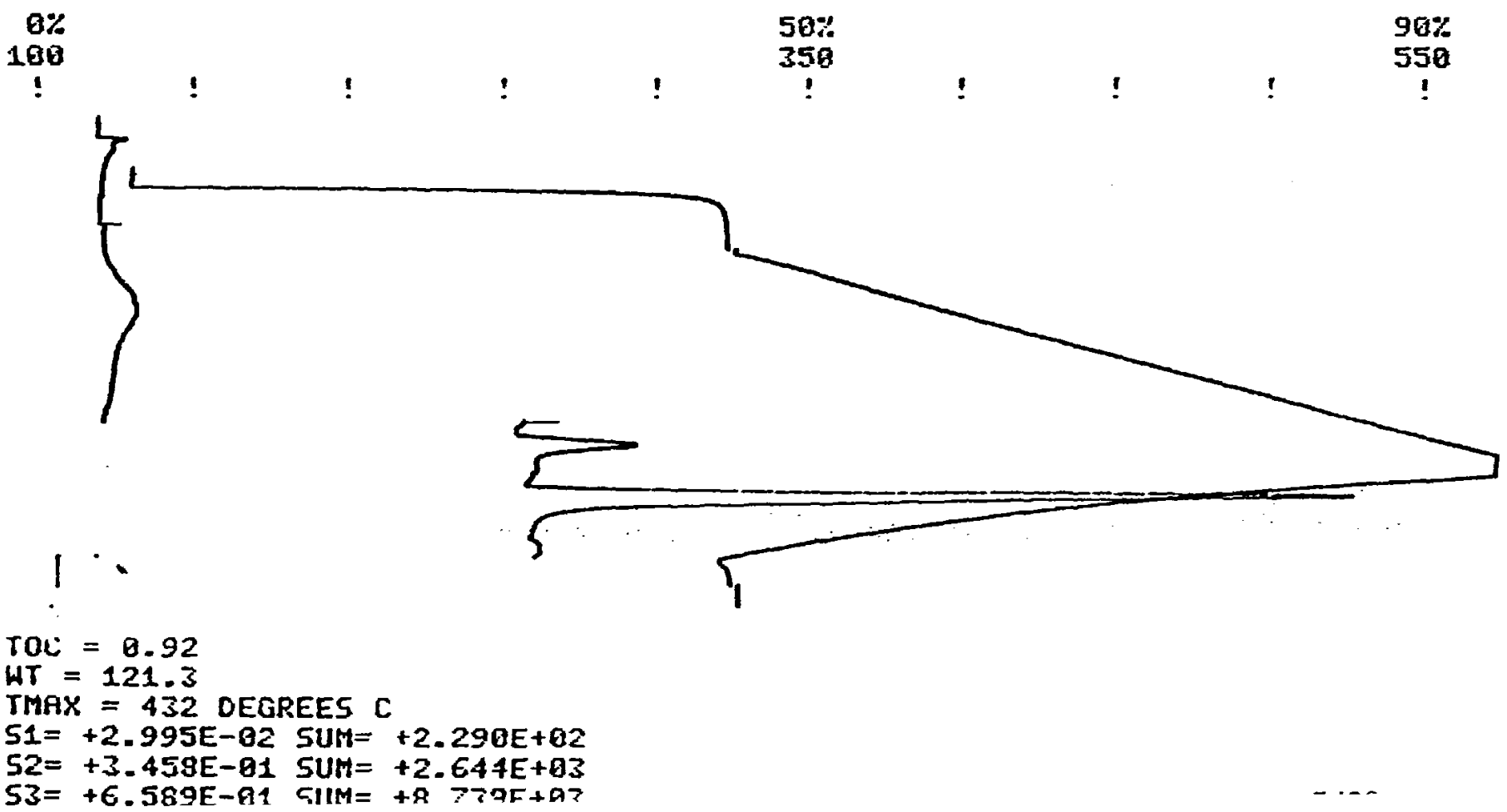


MAY 23, 1985

TIME $=2 \overline{116}$

$\mathrm{IC}=009$

FIU ATTERUATIOH= 32

TCU ATTENUATIOH $=20$

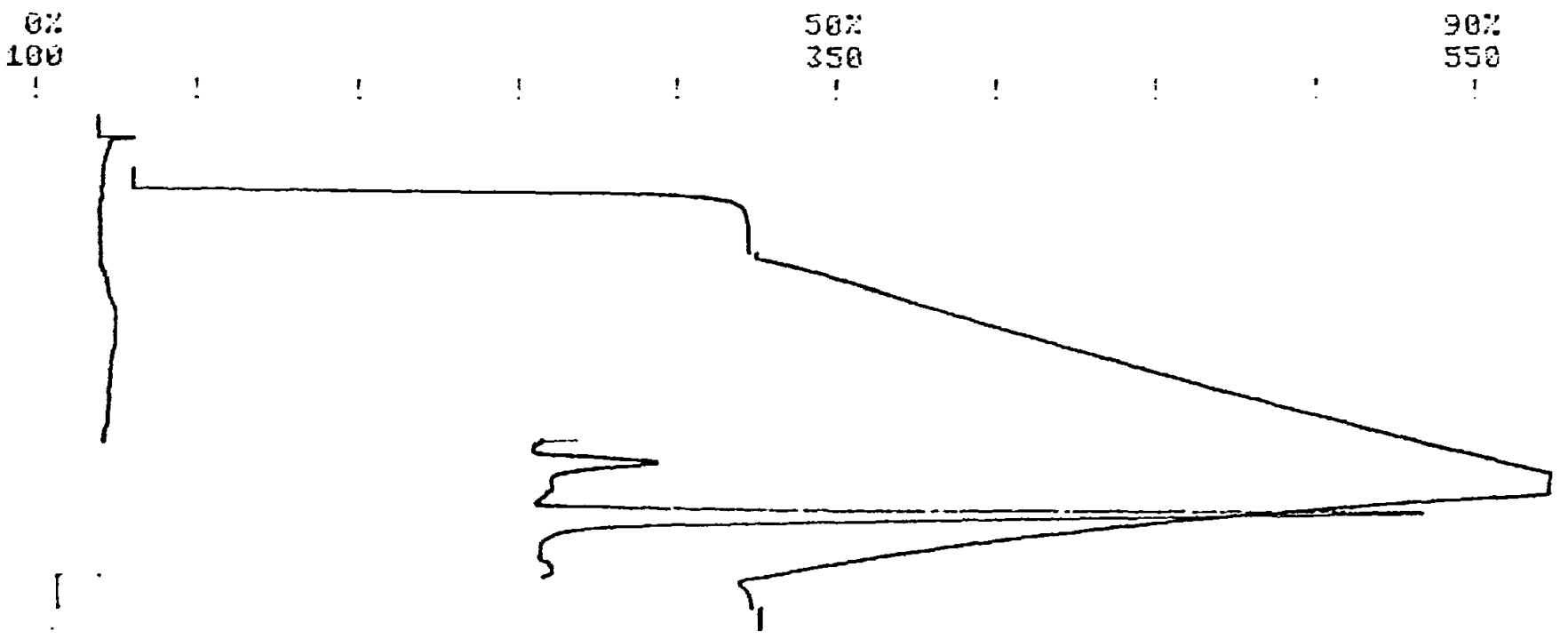

TOL $=0.61$

$H T=120.9$

THAX $=436$ DEGREES $\mathrm{C}$

$S 1=+1.850 E-02$ SUM $=+1.410 E+02$

$52=+1.395 E-01$ SUM $=+1.863 \mathrm{E}+0.3$

$\mathrm{S} J=+6.504 E-81$ JUM $=+8.662 \mathrm{E}+\mathrm{QZ}$

. UNKHOLH

MAY 23, 1928

GEOCOM ROCK EYAL II

TIME $=2043$

$I D=010$

FID ATTENUATIOH $=32$

TCD ATTENUATION= 32

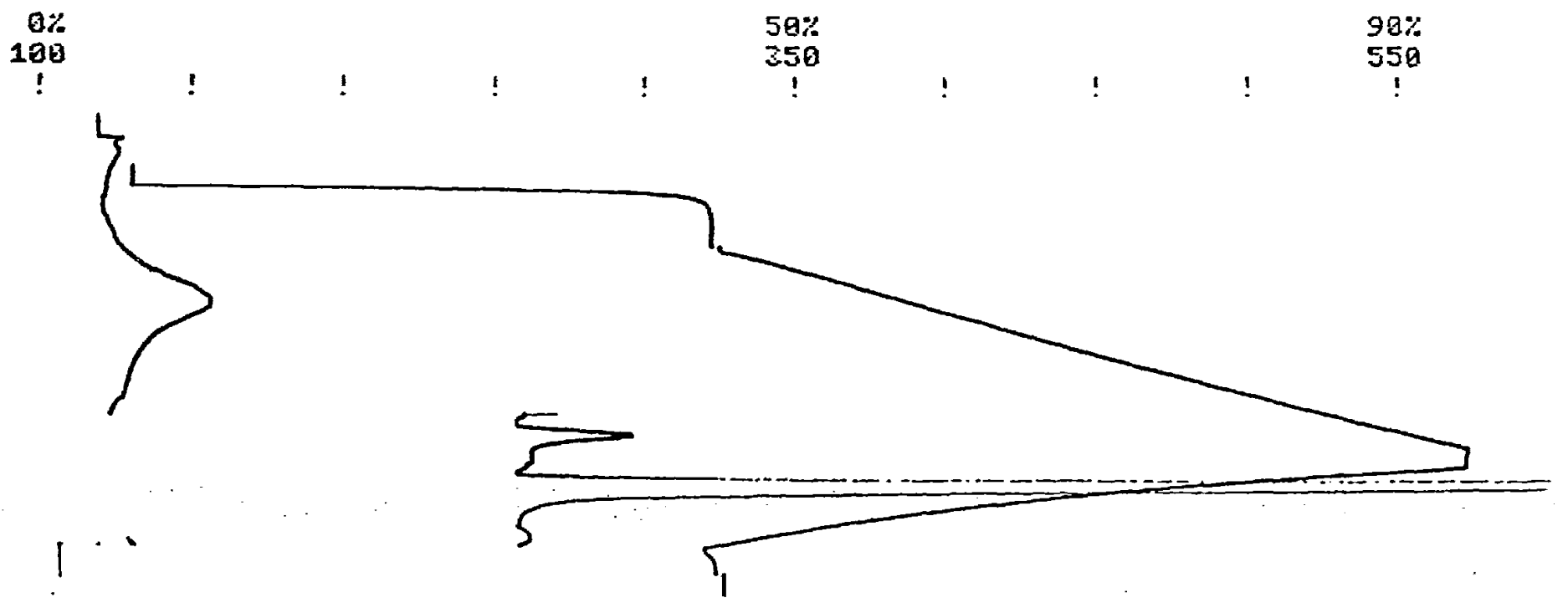

TOC $=1.95$

$W T=113.7$

THAX $=430$ DEGREES $C$

$51=+6.390 E-02$ SUM $=+4.738 E+82$

$52=+1.074 E+00$ SUM $=+8.034 E+03$

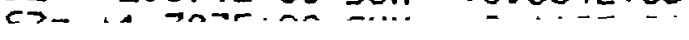




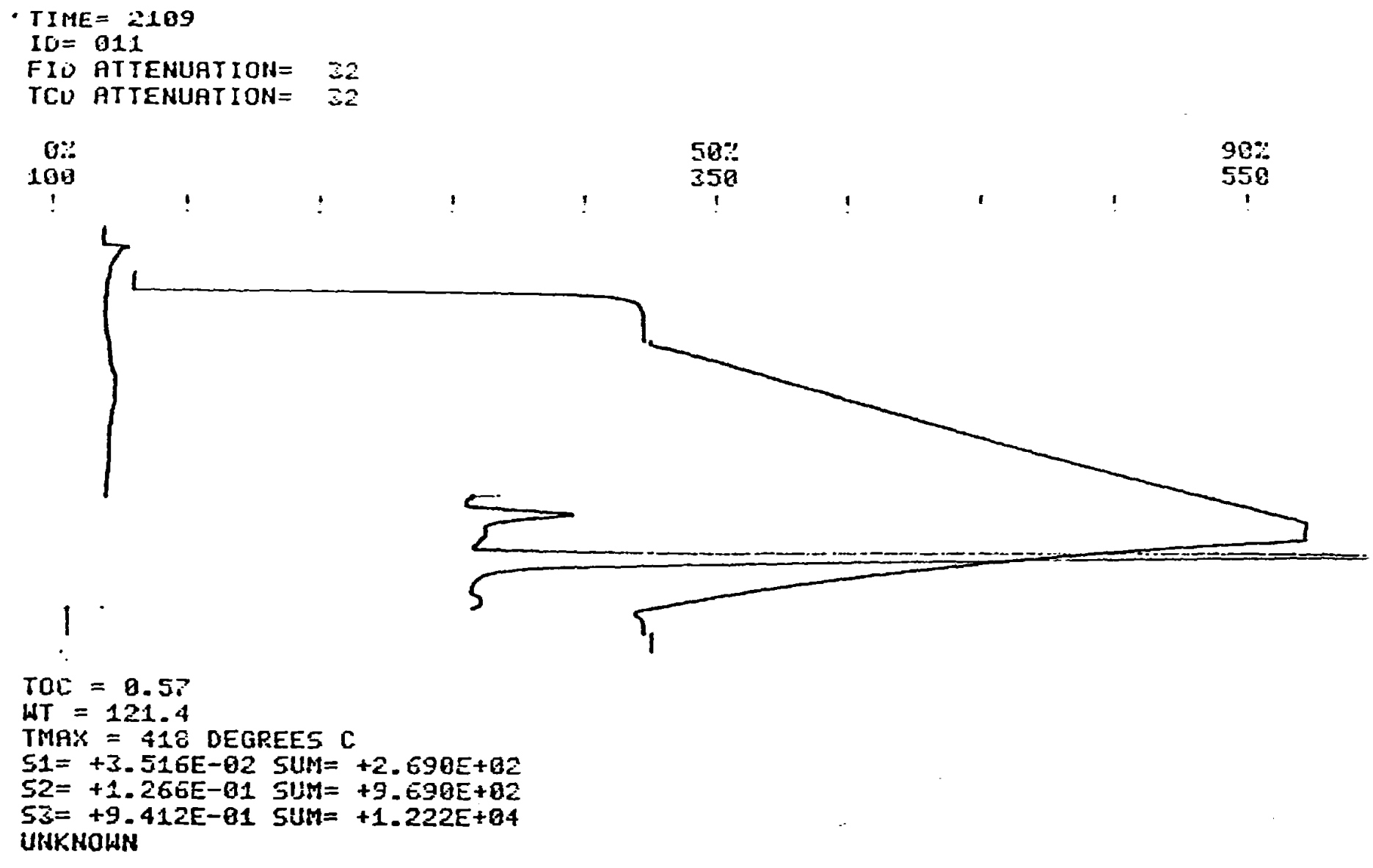

MAI 23, 1988

GECCOH ROCK EVAL II

TIME $=2135$

ID $=012$

FIO ATTENUATIOH $=32$

TCD ATTENUATION= 32

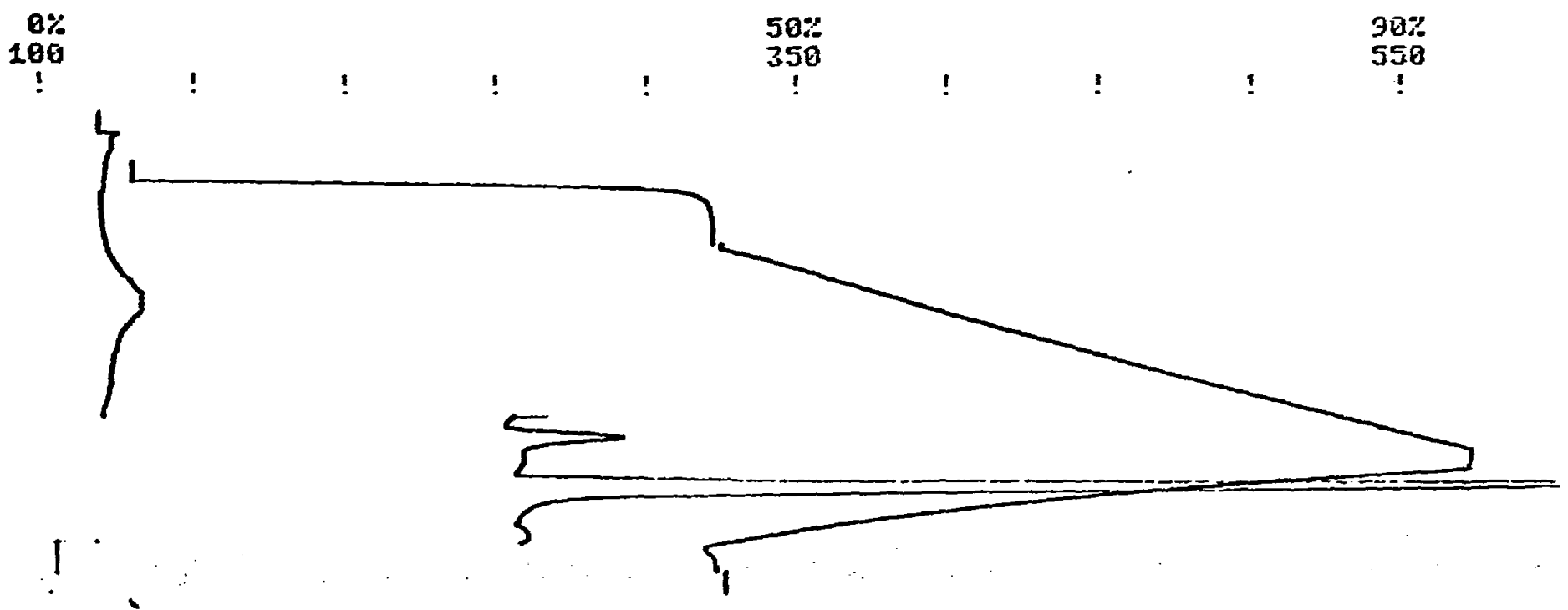

$T Q L=1.00$

$H T=113.5$

TMFX $=428$ DEGREES $C$

$51=+2.921 E-\theta Z$ SUM $=+2.200 E+02$

$S 2=+3.849 E-01$ SUH $=+2.899 E+03$

$S 3=+1.191 \mathrm{E}+00$ SUM $=+1.507 \mathrm{E}+84$

UHKNOWN 
MA' 23,1986

GEOCCM ROCK EYAL II

TIME $=2202$

$I D=613$

FIU ATTENUATIOH= 32

TCU ATTENUATION $=32$

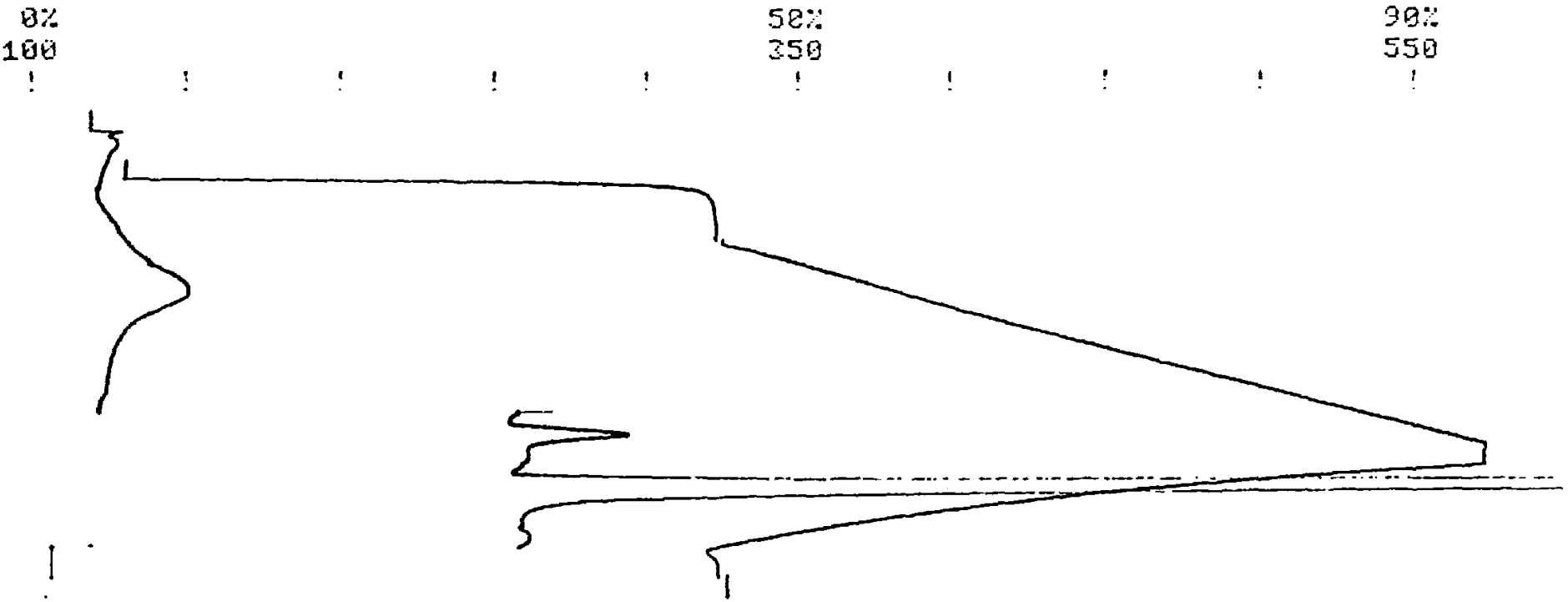

$T a t=1.28$

$\mathrm{HT}=113.3$

THAH $=424$ DEGREES $C$

$51=+9.034 E-02$ SUM $=+6.030 E+02$

$52=+8.847 \mathrm{E}-01$ SUM $=+6.652 \mathrm{E}+63$

$53=+2.265 E+80$ SUM $=+2.805 E+04$

UNKNOWH

MAY 23, 1988

GECCOM ROCK EYAL II

$T$ THE $=2228$

$I D=014$

FID ATTENUATION $=32$

TCD ATTENUATION= 32

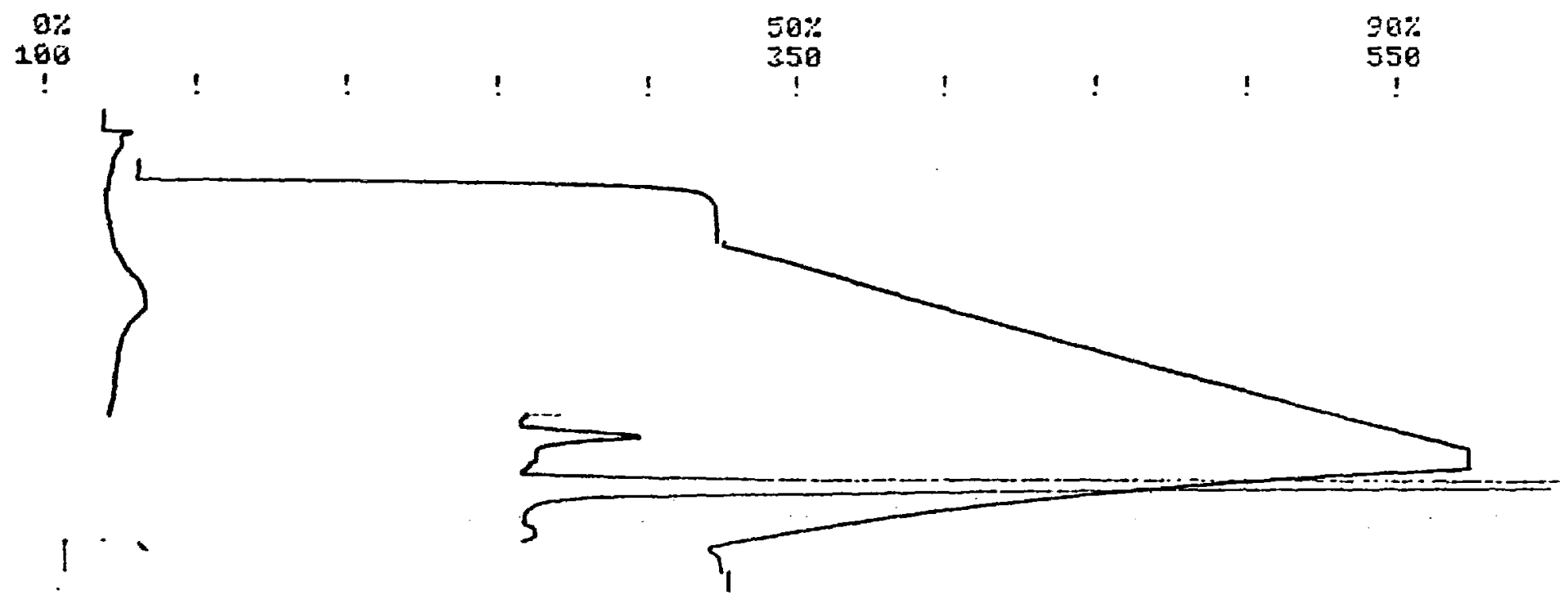

$T O C=1.08$

$H T=121.3$

TMAX $=427$. DEGREES $C$

$51=+4.500 E-02$ SUH $=+3.440 E+02$

$S 2=+3.812 E-B 1 \quad S U H=+2.914 E+03$

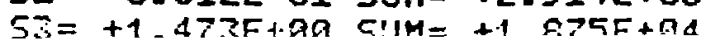




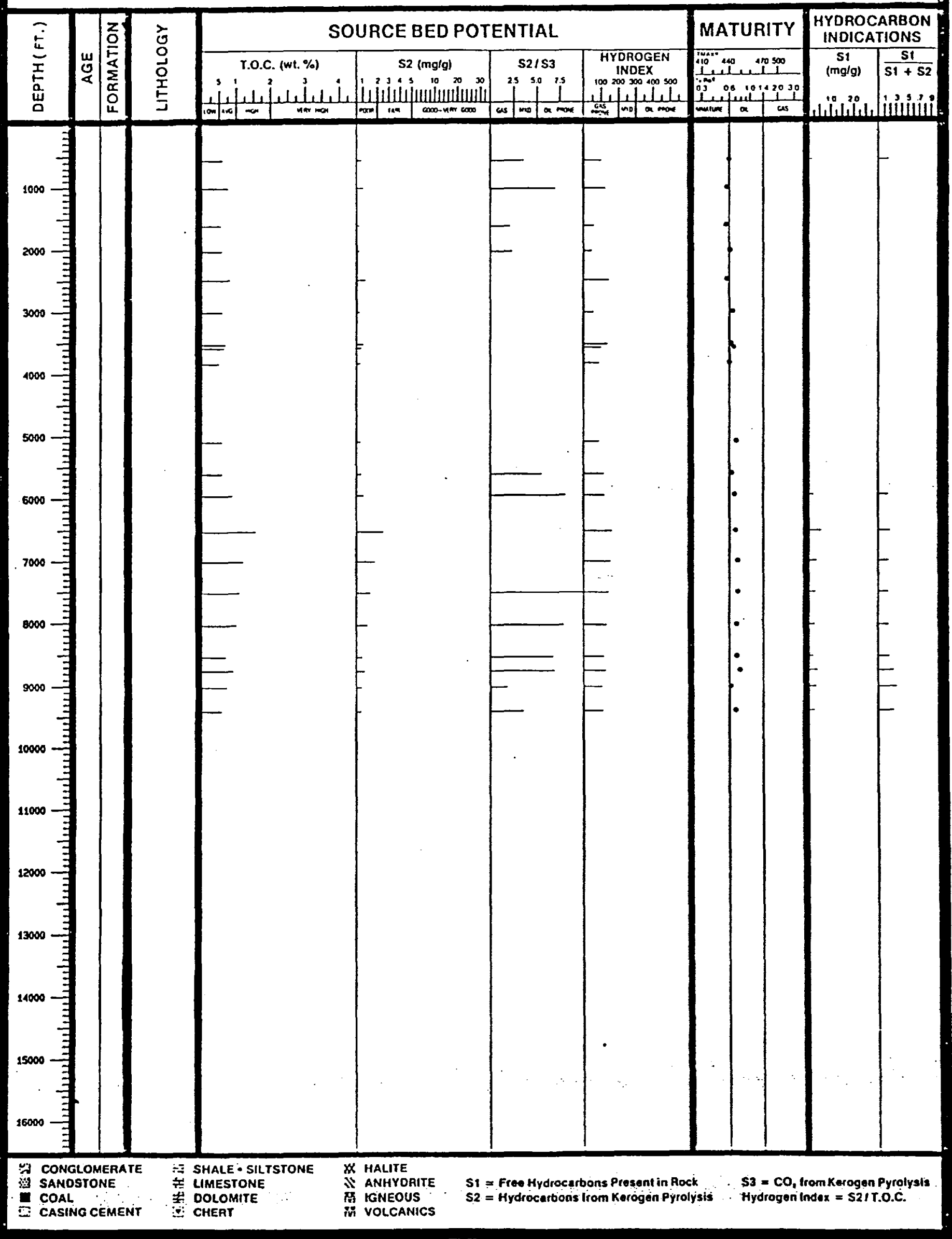


MAY 24,1988

GEOCOM ROCK EVAL II

TIME = 1246

$I D=023$

FIO ATTEHUATION= 32

TCO ATTENUATION $=32$

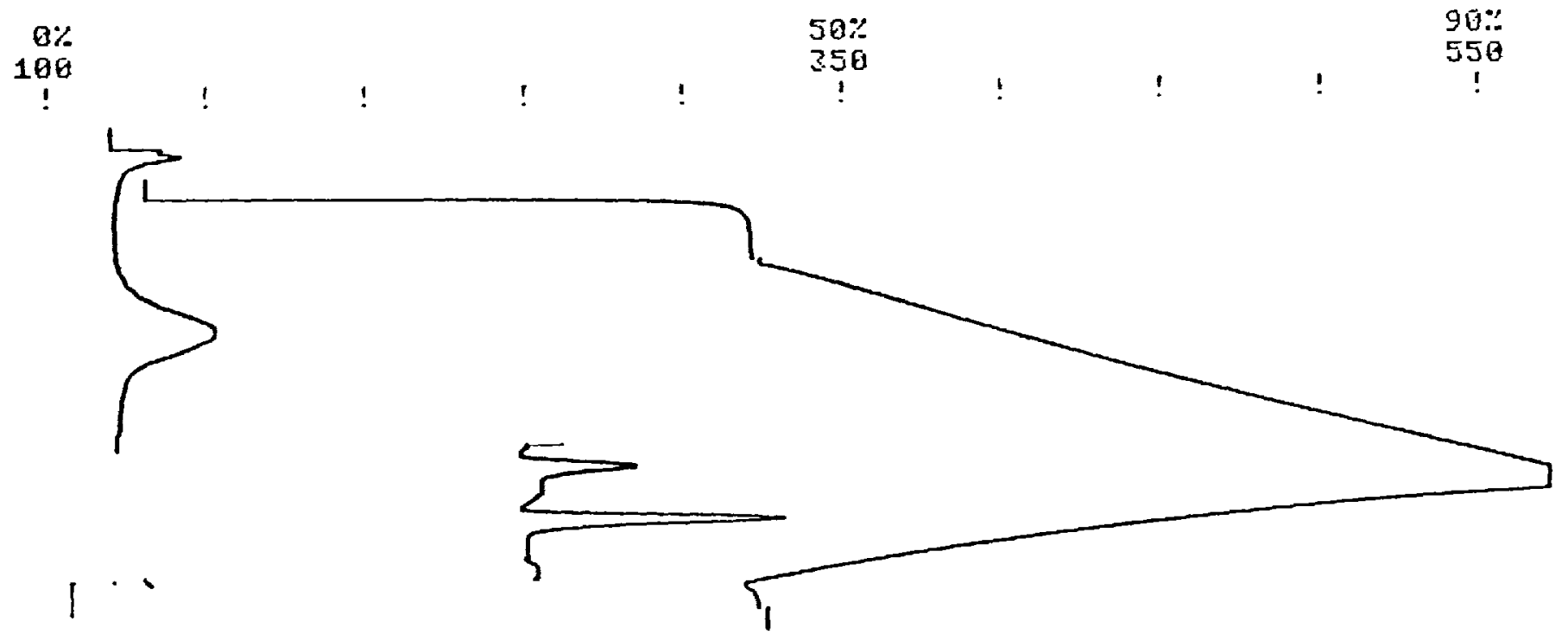

TOC $=0.58$

$W T=120.8$

TMEY $=436$ DEGREES $\mathrm{C}$

$S 1=+9.539 E-02$ SUM $=+7.720 E+02$

$52=+5.576 E-01$ SUM $=+4.508 E+03$

$53=+1.623 E-01$ SUH $=+2.22 E E+03$ 


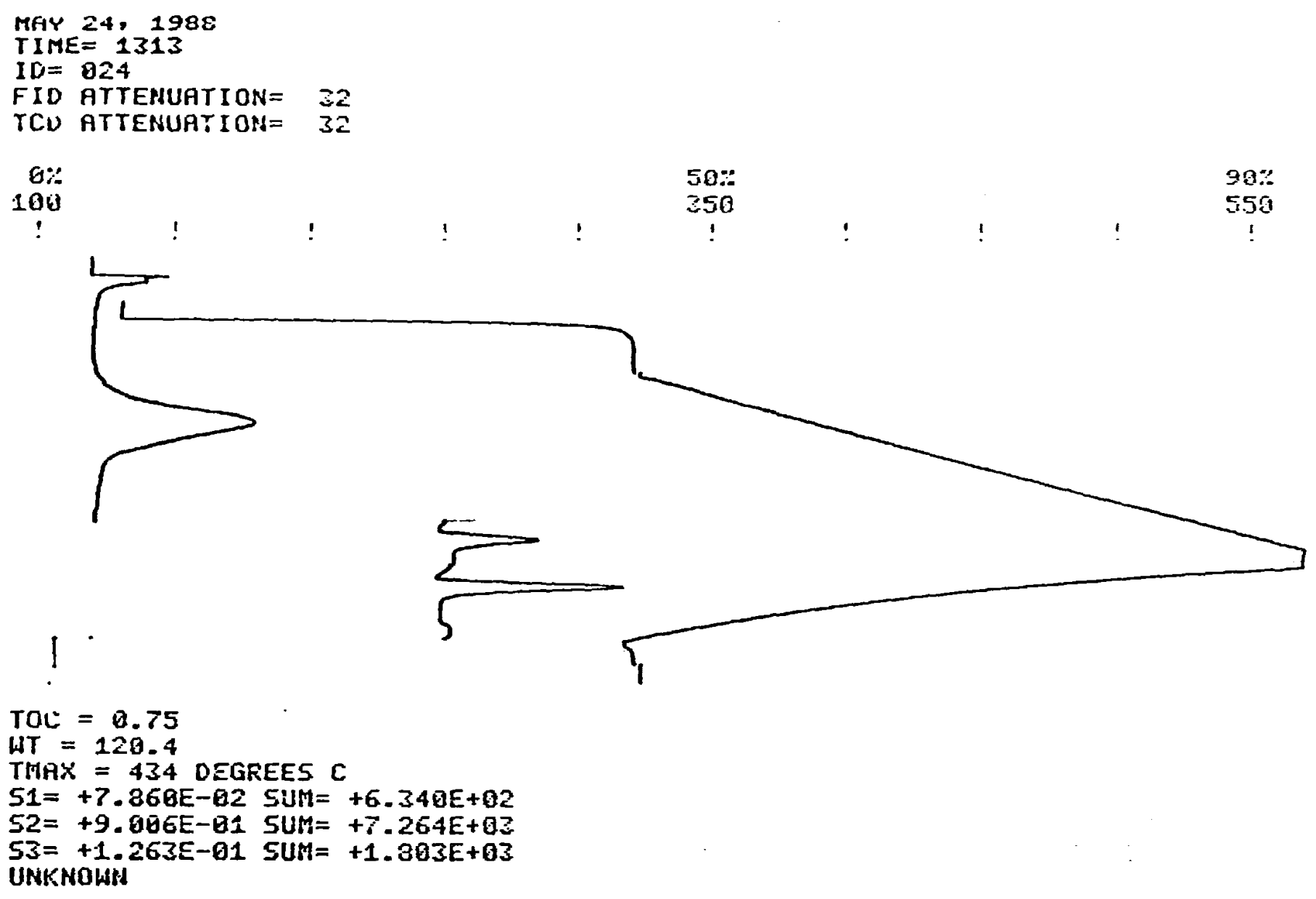

MAY 24, 1989

GEOCOM ROCK EVAL II

TIME $=1339$

ID $=025$

FID ATTENUAT ION= 32

TCO RTTENUAT ION $=32$

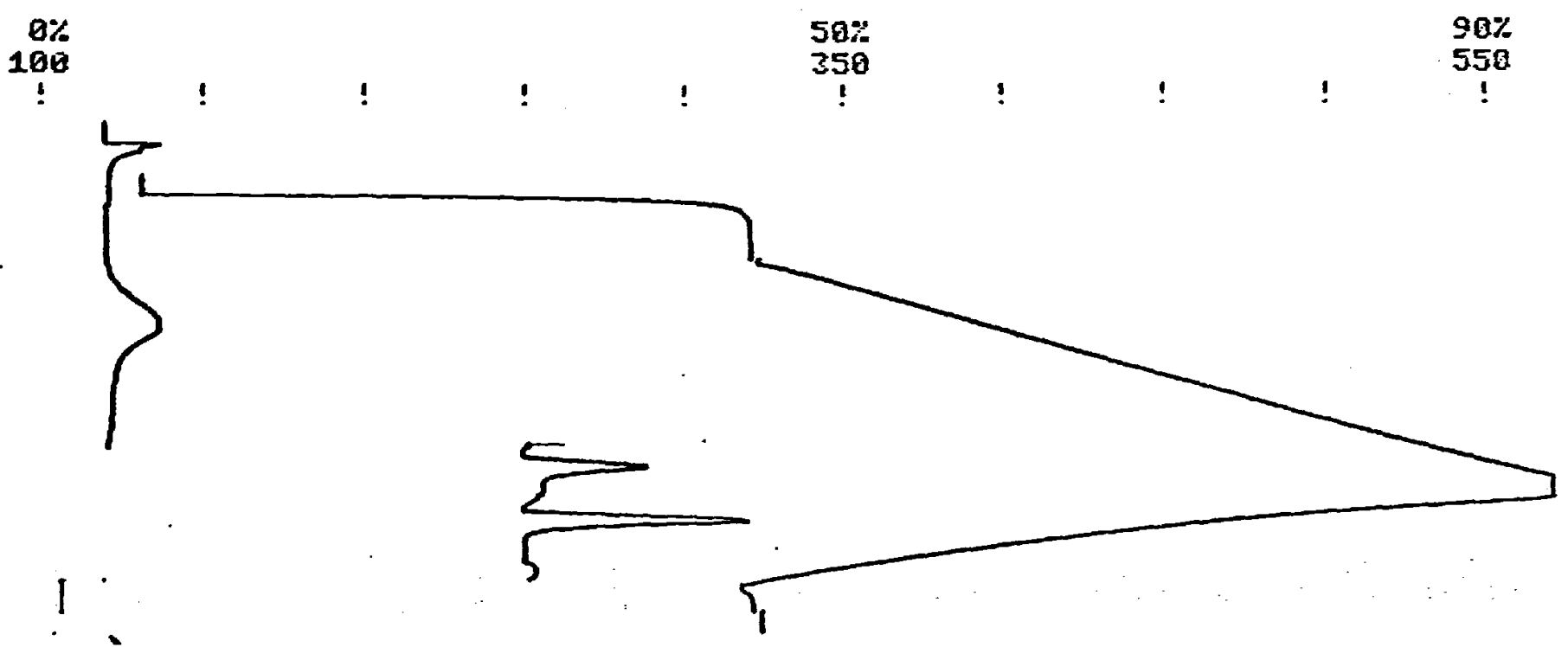

TOC $=0.53$

$H T=120.3$

TMHX $=433$ DEGREES $\mathrm{C}$

$51=+4.510 E-02$ SUM $=+3.650 E+62$

$52=+2.784 E-01$ SUM $=+2.253 E+82$

$53=+1.360 E-01$ SUM $=+1.921 E+03$

UNKHOUNA rMr nats Dannnt 115 
MAY 24,1983

GEOCOM ROCK EVAL I!

TIME $=1405$

IO $=02 \epsilon$

FID ATTENUATION= 22

TCO ATTERUATIOH= 32

$0 \%$

104

$50 \%$

$90 \%$

$!$

350

550

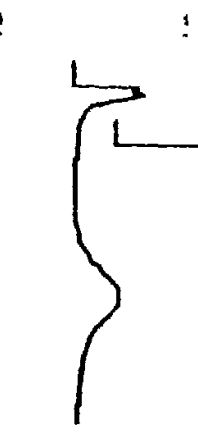

1
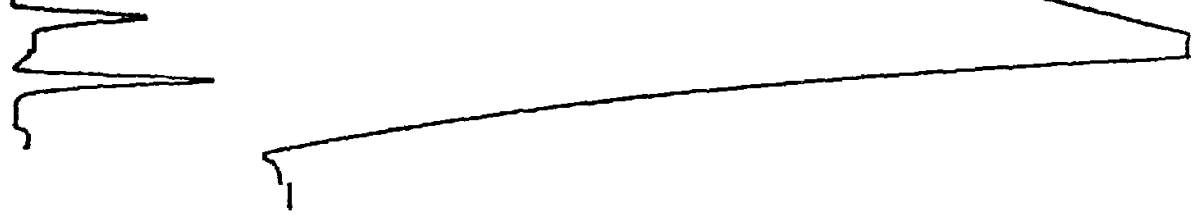

TOL $=0.55$

$H T=120.9$

THAT $=438$ DEGREES $C$

$51=+7.433 E-02$ SUM $=+6.020 E+02$

$52=+2.244 E-01$ SUM $=+1.818 E+83$

$5 \bar{S}=+9.623 \mathrm{E}-82$ SUM $=+1.466 E+03$

UNKRIOWN

MAY 24,1982

GEOCON ROCK EYRL II

TIME $=1431$

$I D=027$

FIO ATTENUATION= 32

TCO ATTENUATION= 32

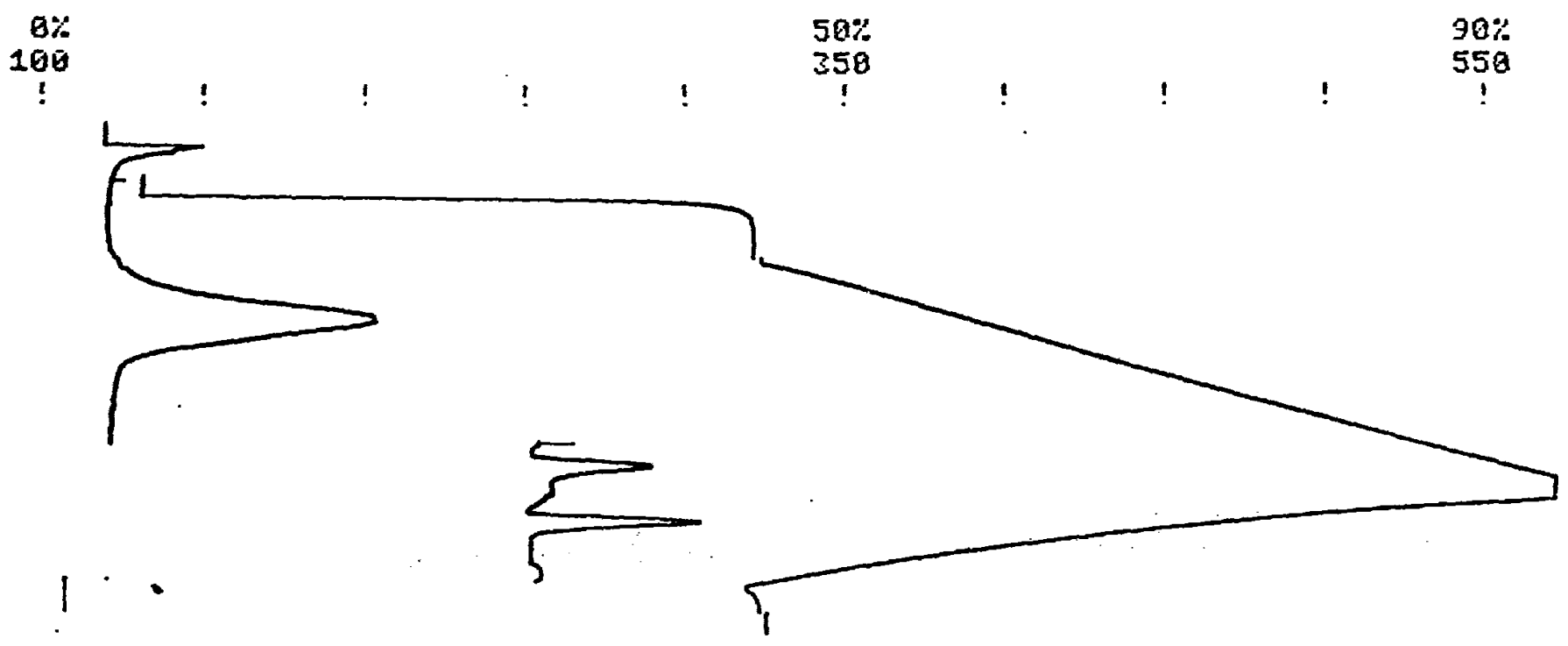

TOC $=0.79$

$H T=123.4$

TMAX $=434$ OEGREES $C$

$51=+3.480 E-02$ SUM $=+7.810 E+82$

$S 2=+1.102 E+60$ SUM $=+9 \cdot 111 E+02$

$53=+8.592 E-62$ SUH $=+1.364 \mathrm{~F}+02$

nur nata Dnomant 115 
MAT 24,1983

TIHE $=1458$

$I D=028$

FIO ATTENUATION $=32$

TCU ATTENUATION= 22

$0:$

$10 \overline{1}$

50

356

$30 \%$

556
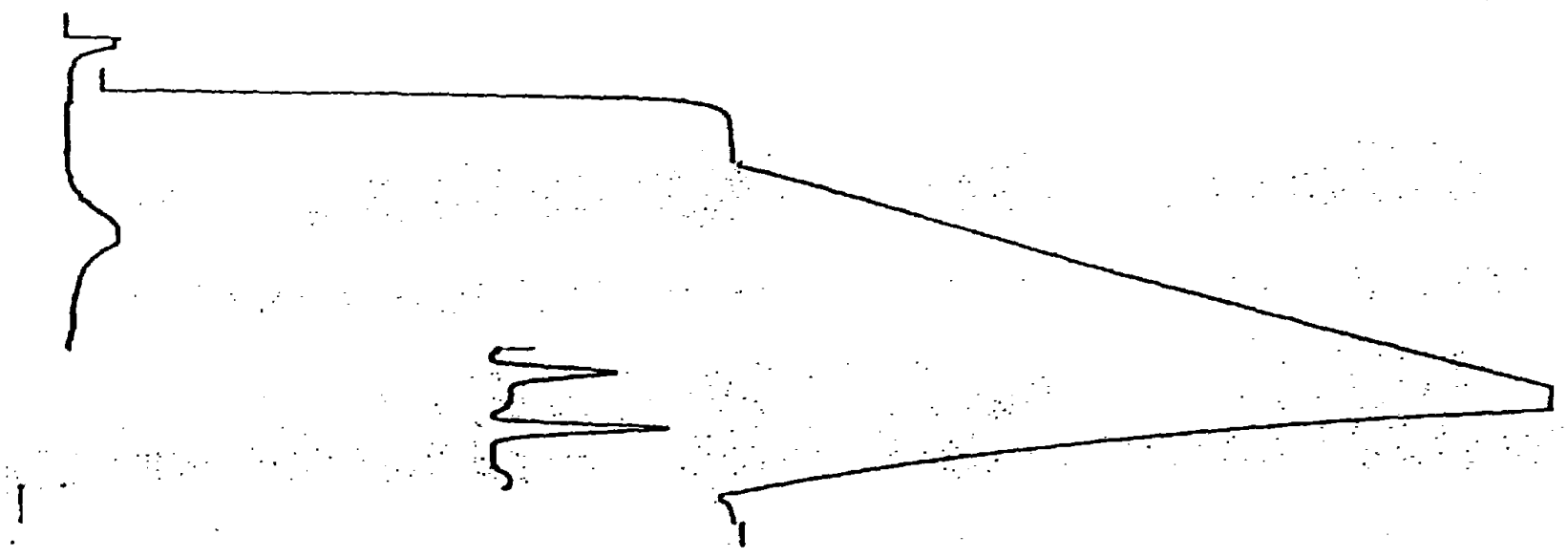

TOC $=0.56$

$H T=124.2$

TMAX $=440$ DEGREES $C$

$51=+6.069 E-02$ SUH $=+5.050 E+02$

$52=+2.799 \mathrm{E}-01 \mathrm{SUH}=+2.329 \dot{\mathrm{E}}+03$

$53=+S \cdot 621 E-025 U M=+1.371 E+03$

UNKNOWH

GMC Data Report 115

GEOCOM POCK EYAL II

Page 15/22

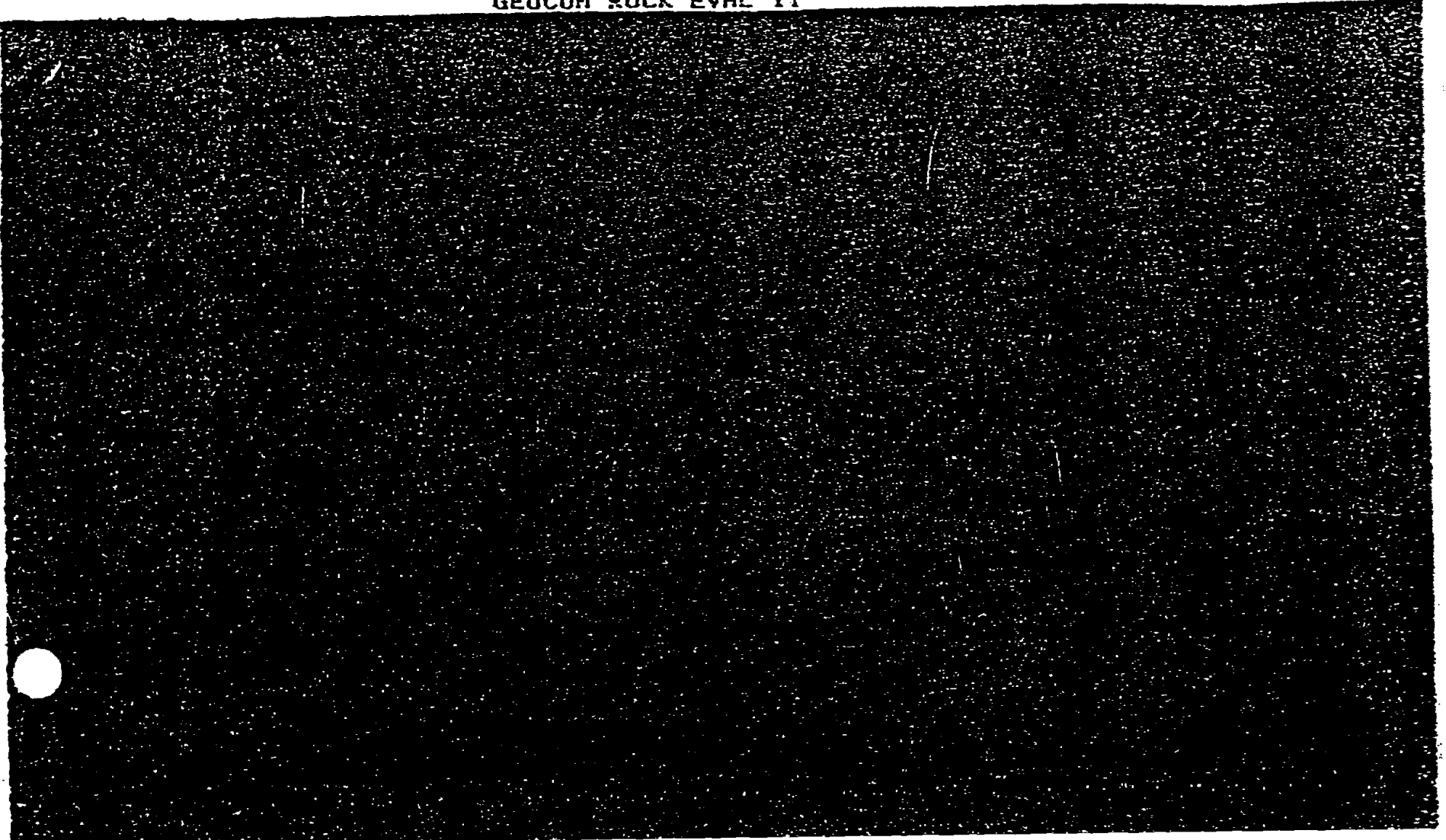


MAY 24,1980

$T I M E=1743$

$I D=38029$

FID ATTENUATION $=32$

TCO ATTENUFTION= 32

Bi:

$10 \overline{0}$

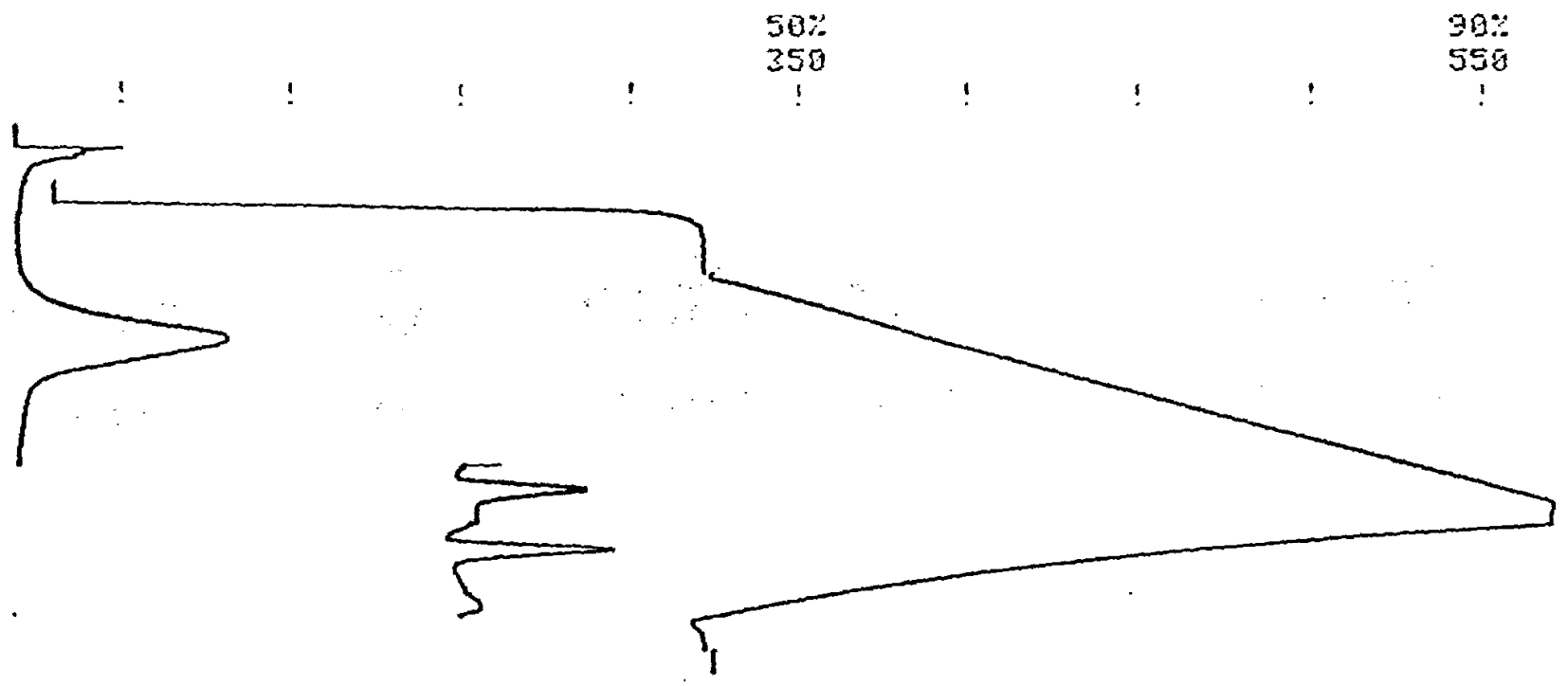

$T O C=0.6 E$

$H T=122.4$

THAY = 433 DEGREES C

$S_{1}=+8.422 E-02$ SUM $=+6.300 E+02$

$52=+3.674 E-61$ SUM $=+7.003 E+02$

$S 3=+7.552 E-02$ SUM $=+1.101 E \div 03$

UNKKIOWN

MAY 24,1988

TIME $=1899$

$I D=030$

FIO ATTENURTION= 32

TCO ATTENUATION= 32

$6 \%$

10

!

GEOCOH ROCK EVRL II
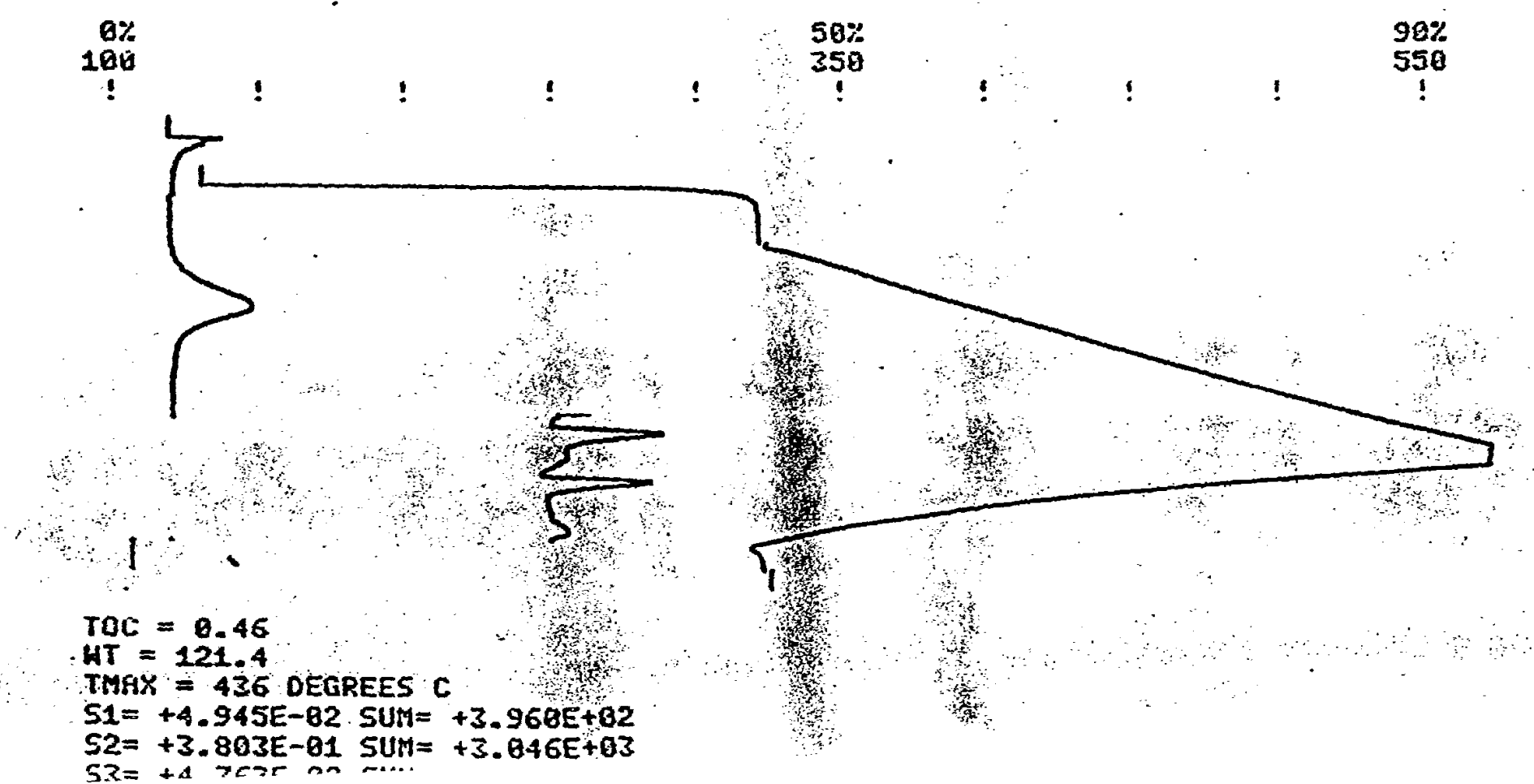
MAY 24, 1983

TI:AE $=1835$

$I D=031$

FIO ATTENUATIOA: $=32$

TCW RTTEHUAT IOH $=32$

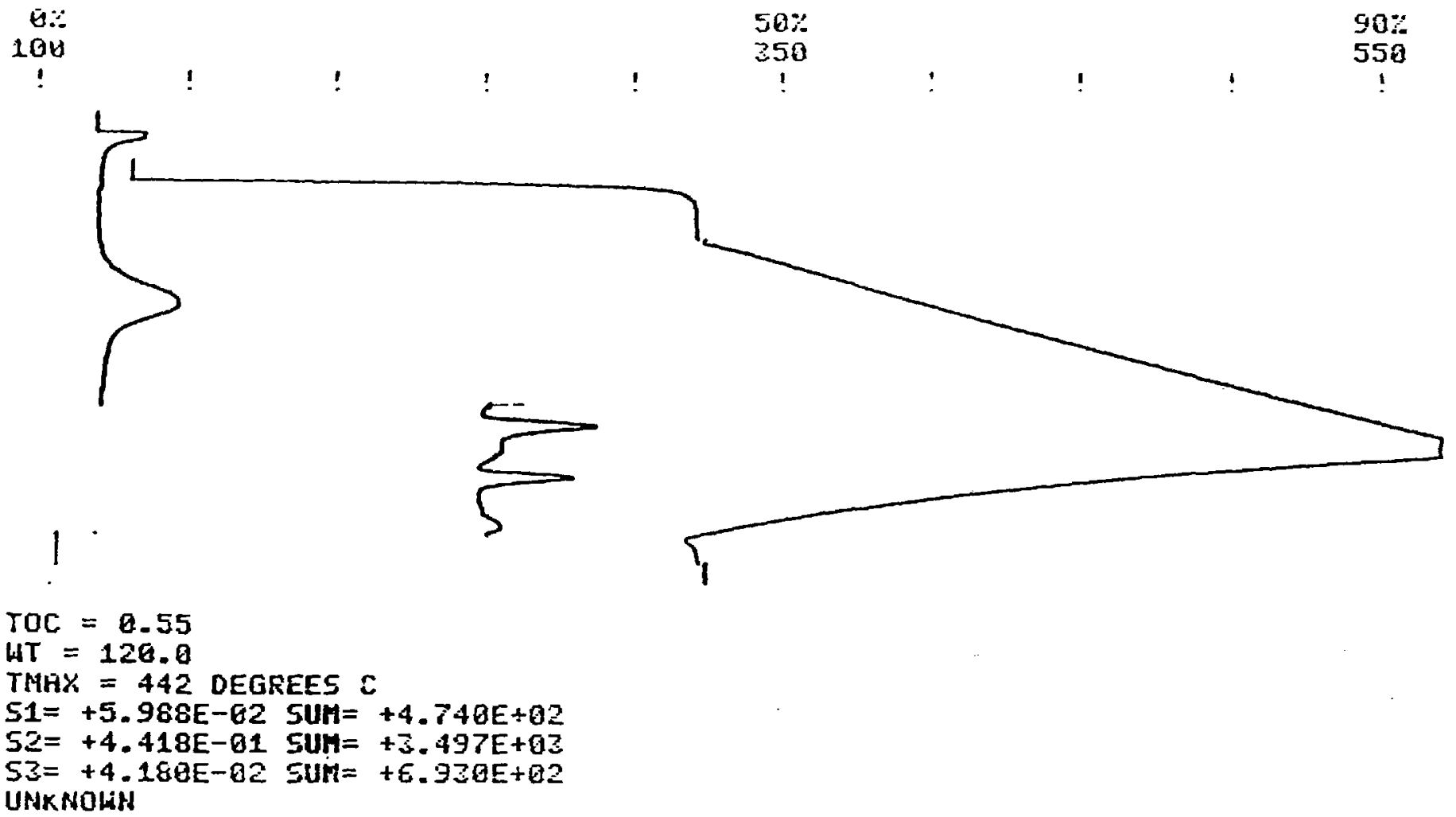

MFY 24, 1988

GECCOM ROCK EYAL II

TIME $=1902$

$I D=G 32$

FID ATTENUATIOH $=32$

TCD ATTENLATIOH= 32

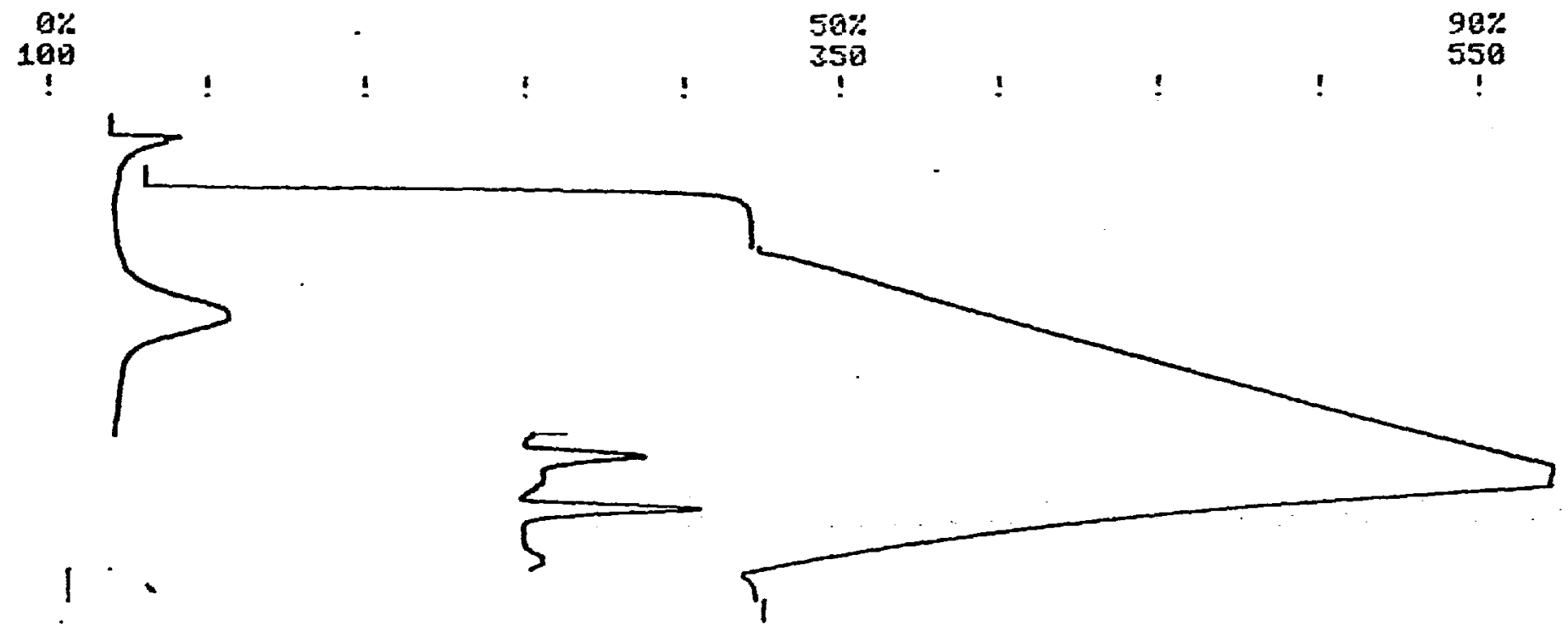

TOC $=0.55$

$4 T=120.7$

TMAX $=438$ DEGREES C

$\mathrm{S} 1=+9.458 \mathrm{E}-02 \mathrm{SUH}=+7.530 \mathrm{E}+02$

$5 z=+5.916 \mathrm{E}-01 \mathrm{SUM}=+4.710 \mathrm{E}+0 \mathrm{z}$

rur $\mathrm{n}_{3}+\mathrm{s}$ Dennent 115

Page $17 / 22$ 
NAY 24,1985

LEOCOH ROCK EVAL II

TI IIE $=1928$

$I D=023$

FID ATTERUATION $=32$

TCD ATTENUATY ON $=32$

$0 \because$

1013

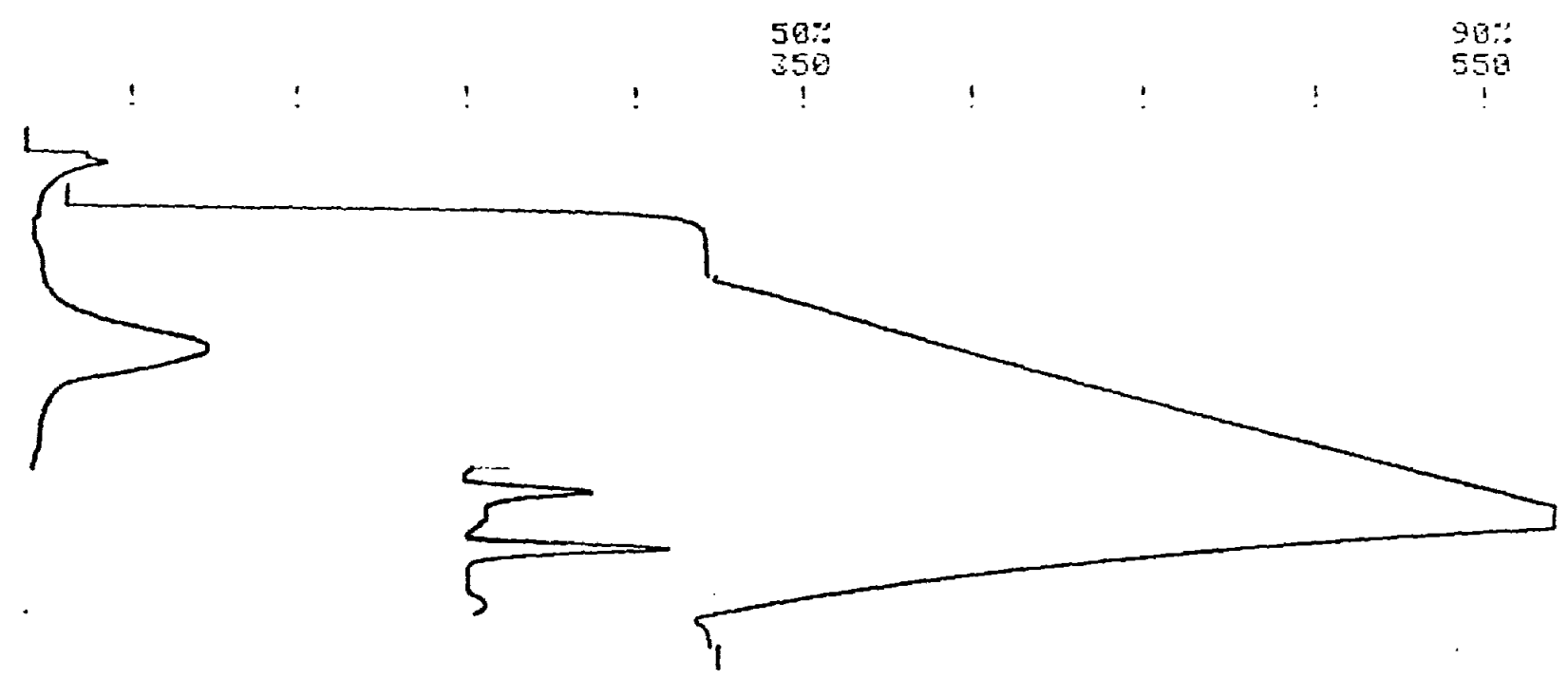

TOL $=0.85$

HT $=123.2$

TMHX $=44 a$ DEGREES $C$

$51=+1.533 E-01$ SUM $=+1.246 \mathrm{E}+63$

$S 2=+9.620 E-01$ SUP $=+? .313 E+03$

$53=+1.193 E-01$ SUI $=+1.623 E+03$

UNKMOHN

MAY 24, 1988

GEOCOM ROCK EVRL II

TIME $=1954$

ID $=634$

FID ATTENUATION $=32$

TCD GTTENUATION= 32

$0 \%$

$50 \%$

$50 \% \quad 98 \%$

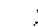

100
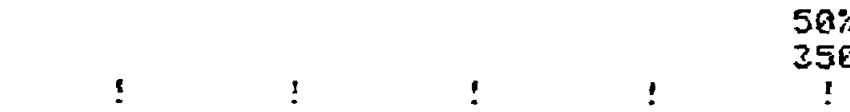

550

(1)

,<smiles>C1CCC1</smiles>
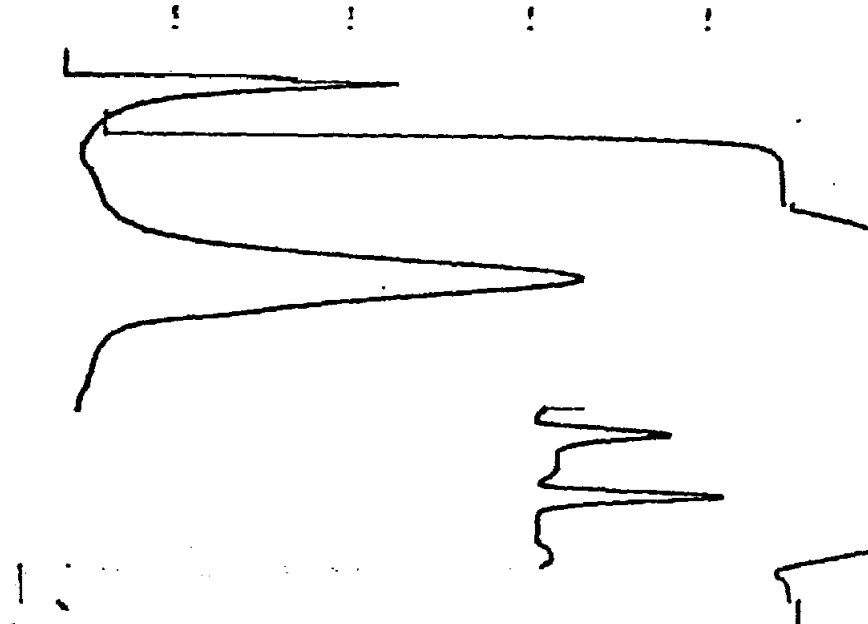

GMC Data Report 115

Page $18 / 22$

$\operatorname{TOC}=1.55$

$H T=120.8$

TMAX $=441$ DEGREES $C$

$S 1=+4.995 E-01$ SUH $=+3.980 E+03$

$52=+2.411 E+0 B$ SUM $=+1.921 E+84$

$S 3=+8.905 E-02$ SUH $=+1.247 E+03$

UNKNOWH 
MfIY 24, 1958

$T I M E=2 B 21$

$I D=035$

FIO ATTENUATION $=32$

TCU FTTENUATION= 32

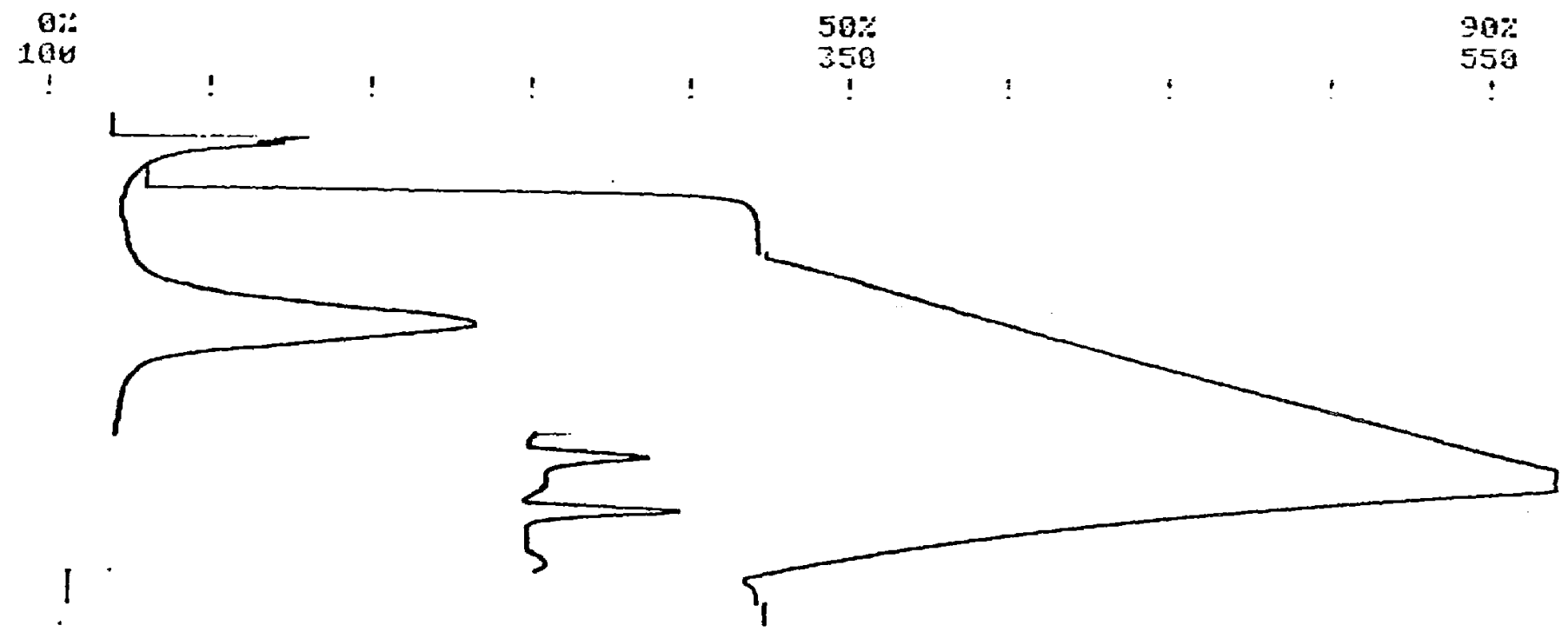

TOC $=1.18$

$W T=121.6$

THAX $=443$ DEGREES $C$

$S_{1}=+3.099 E-01$ SUM $=+2.435 E+83$

$52=+1.755 E+00$ SUM $=+1.407 E+64$

$S 3=+7.662 \mathrm{E}-02$ SUH $=+1.108 \mathrm{E}+02$

UNKHOWN

MAY 24,1928

GECCOM POCK EVAL II

TIME $=2647$

$I D=0 \geq 6$

FID ATTERUATIOH $=32$

TCO ATTEHUATION= 32

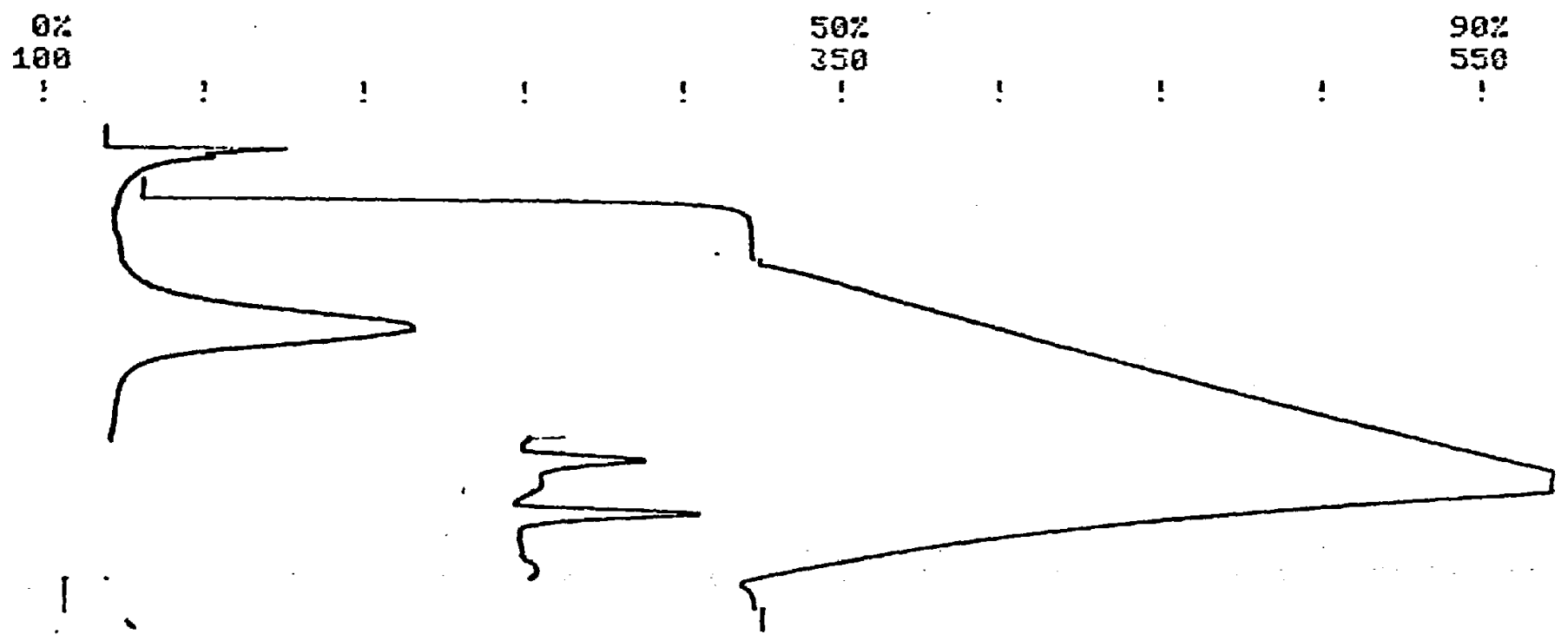

$T O C=1.67$

$W T=120.3$

TMAX $=443$ CEGREES $C$

$S 1=+2.393 E-01 \quad \Xi U M=+1.891 E+03$

$52=+1.441 E+00$ SUM $=+1.143 E+04$

$S 3=+1.013 E-61$ SUT $=+1.391 E+B 3$

GMC Data Report 115

Page $19 / 22$ 
MAY 24,1989

TIME $=2112$

ID $=037$

FIO ATTENUATION $=22$

TCU ATTEHUATION= 32

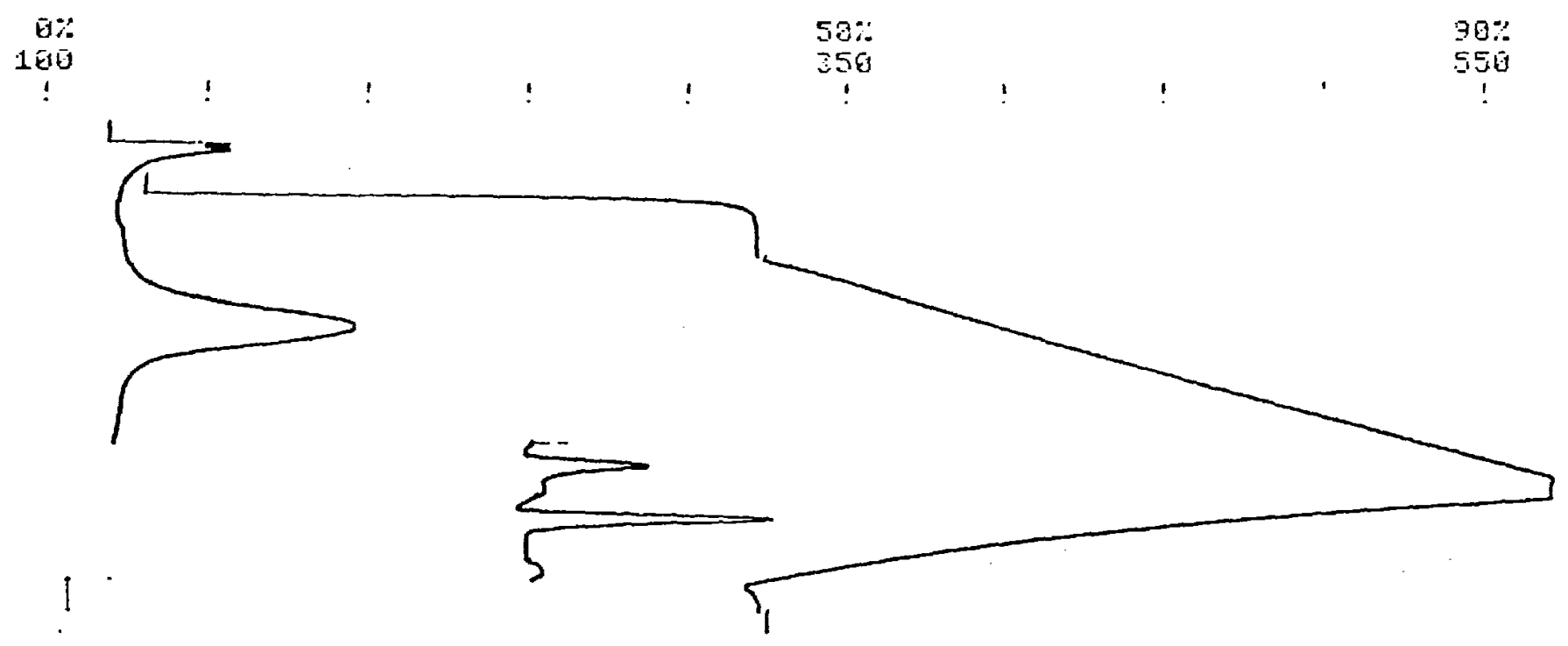

TOC $=0.99$

$4 T=120.5$

TMAX $=442$ DEGREES $C$

$S_{1}=+2.247 \mathrm{E}-01$ SUA $=+1.786 \mathrm{E}+03$

$S 2=+1.245 E+00$ SUM $=+9.8995+33$

$S Z=+1.664 E-01 S U M=+2.074 E+8 Z$

UNKNOWN

MA' 24,1988

GEOCON ROCK EVRL II

TIME $=2140$

$I D=038$

FID RTTENUATION= 32

TCD ATTENUATION= 32

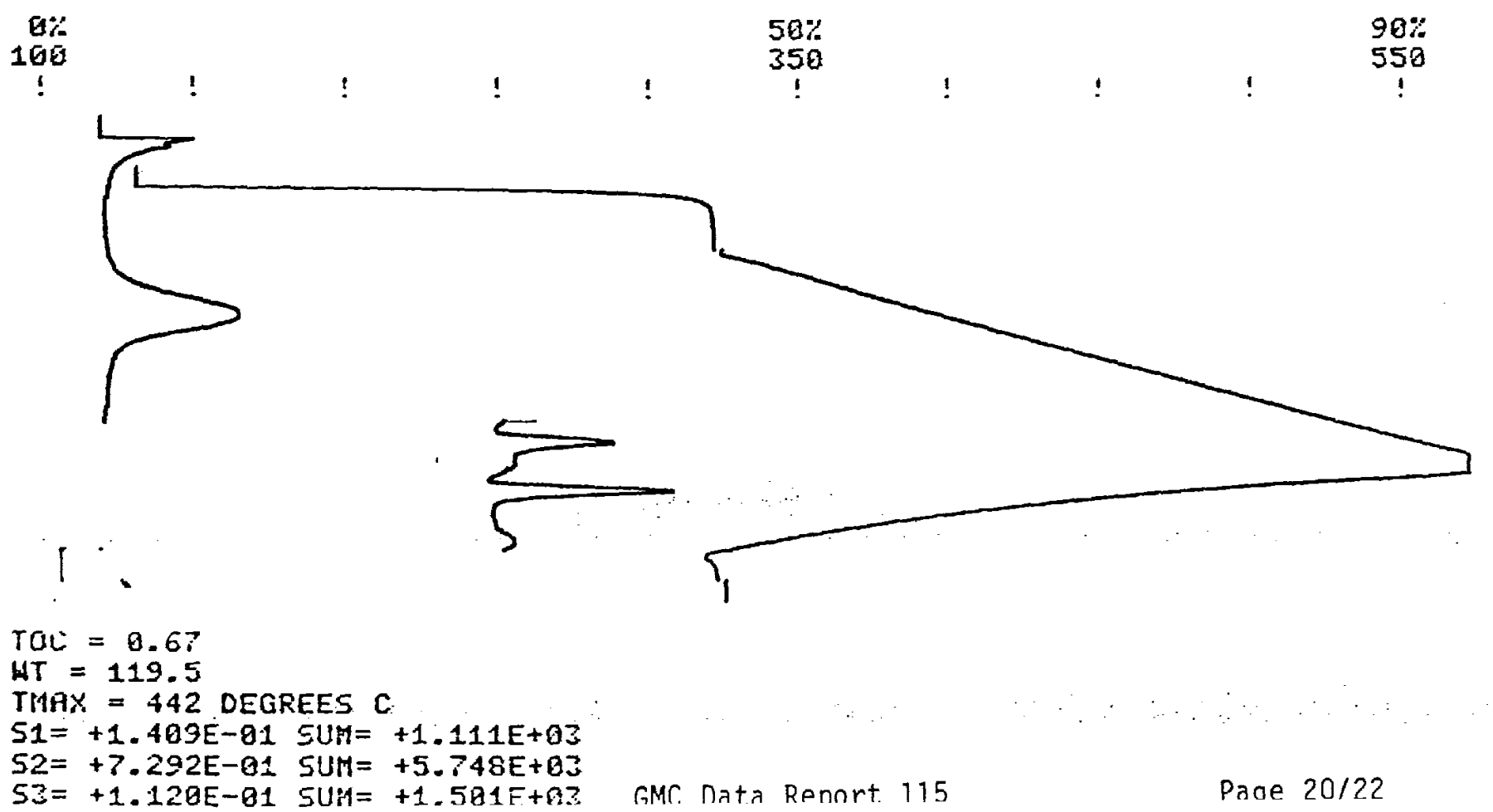


MAY $24,198 \varepsilon$

LEUTUM FUCF, EYAL I:

TIME $=2200^{\circ}$

$I 0=039$

FIU ATTENUATIOH $=22$

TCD ATTENUATIOH= 32

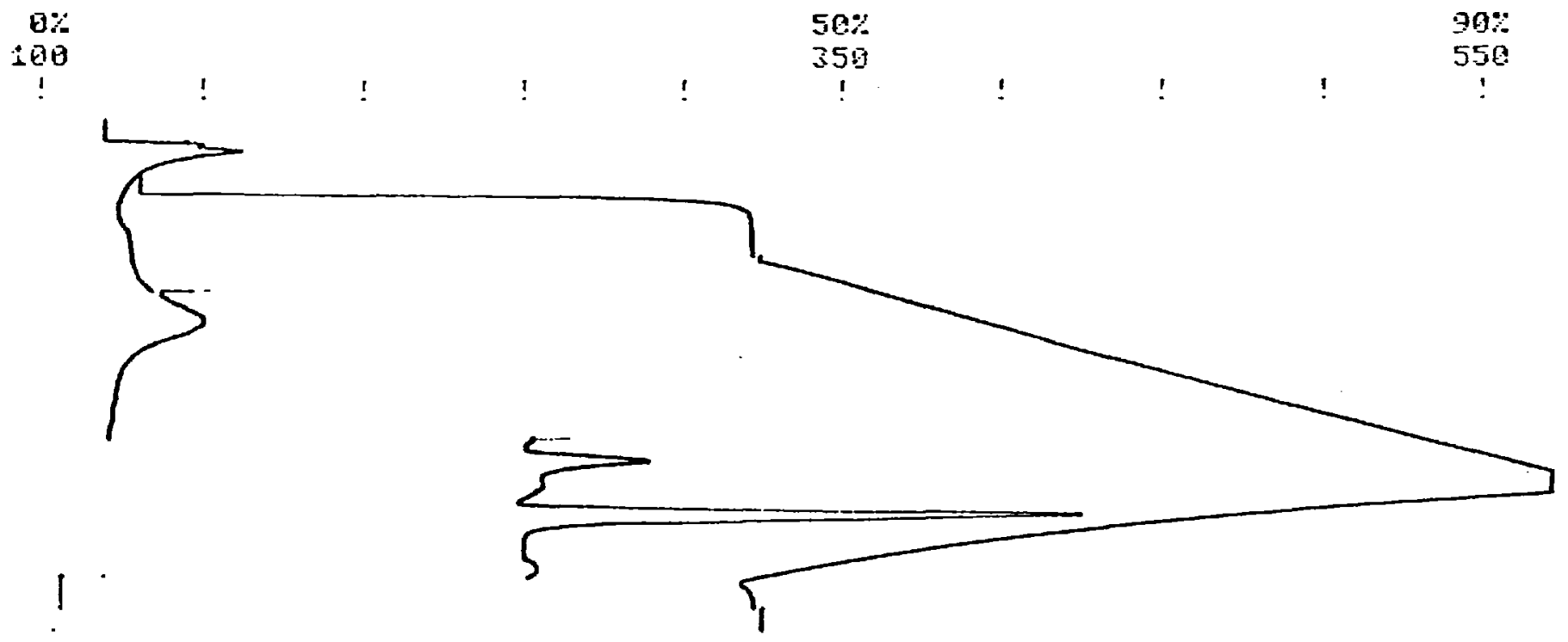

$\operatorname{TOC}=0.78$

$H T=122.0$

TMAX $=437$ DEGREES $C$

$51=+2.369 E-01$ SUT $=+2.261 E+03$

$52=+6.889 E-01$ SUM $=+5.544 E+\theta Z$

$S \bar{S}=+4.156 \mathrm{E}-81 \mathrm{SUM}=+5.102 \mathrm{E}+6 \mathrm{~J}$

UNIKNOWN

MAY 24, 1933

GEOCOM ROCK EYAL II

TIME $=2222$

$I D=040$

FID ATTENUATIOH $=32$

TCO ATTENUATION= 32

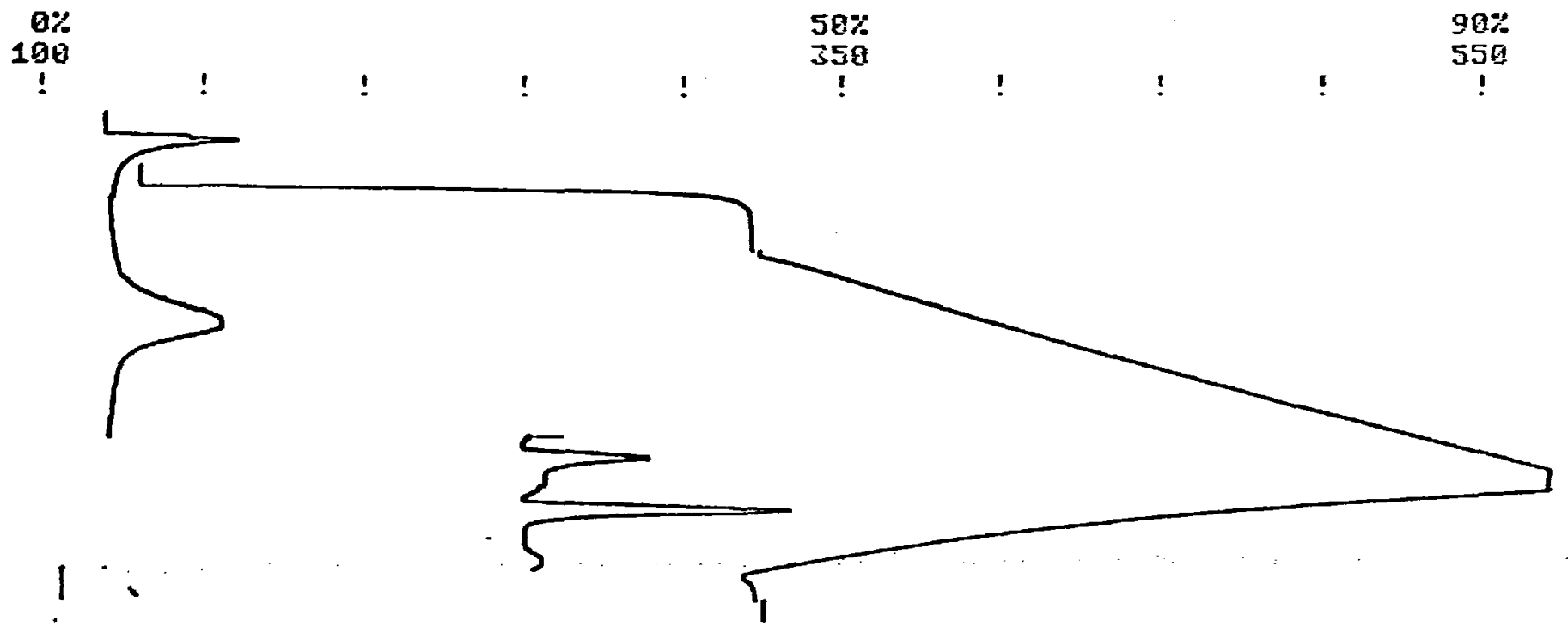

$\mathrm{TOL}=0.55$

$H T=129.5$

TMAX $=441$ OEGREES $\mathrm{C}$

$S 1=+1.836 E-01$ SUM $=+1.46 \theta E+\theta 3$

$S 2=+5.347 E-01$ SUM $=+4.643 E+03$

$S 3=+1.710 E-01$ SUM $=+2.199 E+03$ GMC Data Report 115

Page $21 / 22$ 
VHar 24,1988

TIME $=2258$

$I O=041$

FIO ATTEHUATION= 22

TCL ATTENUATION= 32

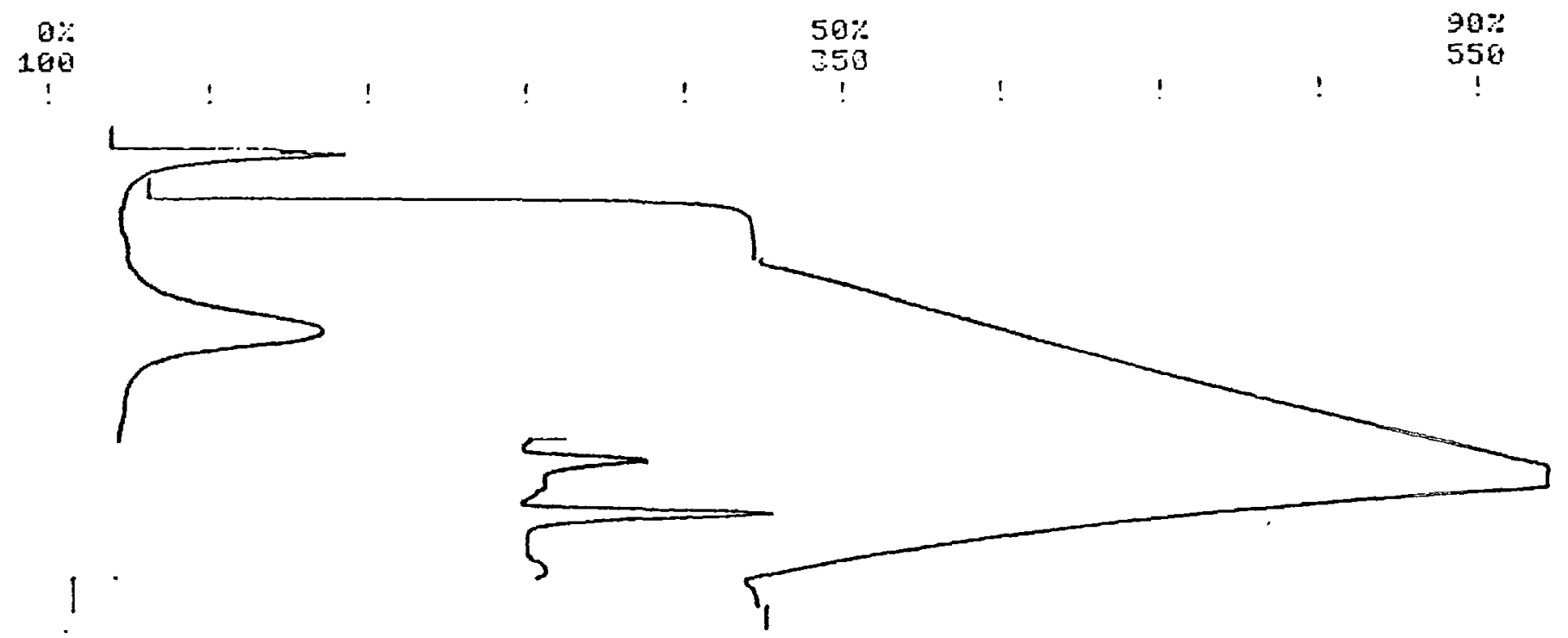

$\mathrm{TOL}=0.90$

$H T=122.5$

TMAX $=445$ OEGREES $C$

$S 1=+3.391 E-01$ SUM $=+2.740 E+B 3$

$52=+1.683 E+60$ SUM $=+8.796 E+83$

$5 \overline{3}=+1.57 \mathrm{PE}-91$ SUN $=+2.674 E+83$

UNKNOWN 